\title{
Regulation of intestinal homeostasis by the ulcerative colitis-associated gene RNF186
}

\author{
Kosuke Fujimoto ${ }^{1,2,3,9}$, Makoto Kinoshita ${ }^{1,2,9}$, Hiroo Tanaka ${ }^{4}$, Daisuke Okuzaki ${ }^{5}$, Yosuke Shimada ${ }^{1,2}$, \\ Hisako Kayama $^{1,2}$, Ryu Okumura ${ }^{1,2}$, Yoki Furuta ${ }^{1,2}$, Masashi Narazaki ${ }^{3}$, Atsushi Tamura ${ }^{4}$, \\ Shigetsugu Hatakeyama ${ }^{6}$, Masahito Ikawa $^{7}$, Kiichiro Tsuchiya $^{8}$, Mamoru Watanabe $^{8}$, \\ Atsushi Kumanogoh ${ }^{2,3}$, Sachiko Tsukita ${ }^{2,4}$ and Kiyoshi Takeda ${ }^{1,2}$
}

Genome-wide association studies and subsequent deep sequencing analysis have identified susceptible loci for inflammatory bowel diseases (IBDs) including ulcerative colitis (UC). A gene encoding RING finger protein 186 (RNF186) is located within UC-susceptible loci. However, it is unclear whether RNF186 is involved in IBD pathogenesis. Here, we show that RNF186 controls protein homeostasis in colonic epithelia and regulates intestinal inflammation. RNF186, which was highly expressed in colonic epithelia, acted as an E3 ligase mediating polyubiquitination of its substrates. Permeability of small organic molecules was augmented in the intestine of $R n f 186^{-I^{-}}$mice. Increased expression of several RNF186 substrates, such as occludin, was found in $R n f 186^{-1-}$ colonic epithelia. The disturbed protein homeostasis in Rnf186 ${ }^{-1-}$ mice correlated with enhanced endoplasmic reticulum (ER) stress in colonic epithelia and increased sensitivity to intestinal inflammation after dextran sulfate sodium (DSS) treatment. Introduction of an UC-associated Rnf186 mutation led to impaired E3 ligase activity and increased sensitivity to DSS-induced intestinal inflammation in mice. Thus, RNF186 maintains gut homeostasis by controlling ER stress in colonic epithelia.

\section{INTRODUCTION}

Inflammatory bowel diseases (IBDs), represented by Crohn's disease (CD) and ulcerative colitis (UC), are chronic inflammatory disorders of the gastrointestinal tract. ${ }^{1}$ Both genetic and environmental factors have a central role in the pathogenesis of $\mathrm{IBD}^{2,3}$ Recent genome-wide association studies and subsequent meta-analyses of IBD patients have identified more than 200 IBD risk loci. ${ }^{4,5}$ Among them, some loci are specific to the risk for either CD or UC. ${ }^{4-8}$ Further analyses of UC-specific loci in UC patients have identified a genetic variation of RNF186 in which alanine at 64 position is substituted with threonine (A64T). ${ }^{9}$ RNF186 is a member of the large RING finger protein family of which many possess an E3 ubiquitin ligase (RING finger E3) activity. ${ }^{10,11}$ RING finger E3s, which comprise the largest subgroup of E3 ligases, have been shown to control many cellular processes including regulation of endoplasmic reticulum (ER) stress. ${ }^{12}$

Several lines of evidence suggest a close association between ER stress and intestinal inflammation. ${ }^{13}$ Increased expression of ER stress markers is observed in IBD patients. ${ }^{14,15}$ Missense mutations of the Muc2 gene, which causes accumulation of Muc2 protein in goblet cells, lead to ER stress and spontaneous development of colitis in mice, indicating that the intestinal inflammation is initiated by ER stress. ${ }^{14}$ A mutation in Mbtps1 (encoding membrane-bound transcription factor peptidase site 1) leads to impairment of the unfolded protein response (UPR) against ER stress and increases the susceptibility to dextran sulfate sodium (DSS)-induced colitis. ${ }^{16}$

\footnotetext{
${ }^{1}$ Laboratory of Immune Regulation, Department of Microbiology and Immunology, Graduate School of Medicine, WPI Immunology Frontier Research Center, Osaka University, Suita, Osaka, Japan. ${ }^{2}$ Core Research for Evolutional Science and Technology, Japan Agency for Medical Research and Development, Tokyo, Japan. ${ }^{3}$ Department of Respiratory Medicine, Allergy and Rheumatic Diseases, Graduate School of Medicine, Osaka University, Suita, Osaka, Japan. ${ }^{4}$ Laboratory of Biological Science, Graduate School of Frontier Biosciences and Graduate School of Medicine, Osaka University, Suita, Osaka, Japan. ${ }^{5}$ DNA-Chip Development Center for Infectious Diseases, Research Institute for Microbial Diseases, Osaka University, Suita, Osaka, Japan. ${ }^{6}$ Department of Biochemistry, Graduate School of Medicine, Hokkaido University, Hokkaido, Japan. ${ }^{7}$ Research Institute for Microbial Diseases, Osaka University, Suita, Osaka, Japan and ${ }^{8}$ Department of Gastroenterology and Hepatology, Tokyo Medical and Dental University, Tokyo, Japan. Correspondence: K Takeda (ktakeda@ongene.med.osaka-u.ac.jp)

${ }^{9}$ These authors contributed equally to this work.
} 
Disruption of the Xbp1 gene, which encodes X-box binding protein 1 (a known UPR transcription factor), in intestinal epithelial cells results in development of spontaneous intestinal inflammation in association with a loss of Paneth cells and goblet cells in mice. ${ }^{15}$ Moreover, intestinal inflammation of IBD patients is strongly correlated with single nucleotide polymorphisms (SNPs) in the XBP1 locus. ${ }^{15}$ These studies indicate that defective responses to ER stress trigger the development of intestinal inflammation.

RNF186 has been shown to mediate the ER stress-induced response. ${ }^{17}$ In addition, hepatocyte nuclear factor $4 \alpha$ (HNF4 $\alpha$ ), which regulates the expression of RNF $186,{ }^{9}$ is associated with intestinal permeability and inflammation. ${ }^{18}$ However, it is unclear whether RNF186 is functionally involved in the pathogenesis of intestinal inflammation.

In this study, we analyzed the role of RNF186 in the maintenance of intestinal homeostasis. RNF186 acted as an E3 ubiquitin ligase that was highly expressed in colonic epithelial cells. In the absence of Rnf186, expression patterns of RNF186 substrates were altered in colonic epithelial cells. Dysregulated protein homeostasis correlated with enhanced ER stress in colonic epithelial cells and high susceptibility to intestinal inflammation after DSS treatment in $R n f 186^{-1-}$ mice. Introduction of the UC-associated A64T mutation in Rnf186 led to impaired E3 ligase activity and an increased risk of intestinal inflammation. These findings suggest that RNF186 is responsible for the maintenance of intestinal homeostasis through the control of protein homeostasis in colonic epithelia.

\section{RESULTS}

\section{Hyperlucency of $R_{n f 186^{-I}}$ colonic epithelia}

RNF186 has been shown to be highly expressed in human intestine. ${ }^{19}$ Therefore, we first analyzed tissue expression of Rnf186 in C57BL/6 J mice. The highest Rnf186 expression was observed in the large intestine (Figure 1a). We then analyzed expression of Rnf186 in the lamina propria and epithelium of the large intestine. Rnf186 was predominantly expressed in the epithelium of the large intestine (Figure 1b). In addition, comparison of RNF186 expression between healthy controls and UC patients in datasets (GSE36807 and GSE38713) deposited in Gene expression Omnibus (http:// www.ncbi.nlm.nih.gov/geo/) showed that RNF186 expression in colonic specimens was significantly decreased in UC patients (Supplementary Figure 1). To assess the physiological function of RNF186, we generated $R n f 186^{-1-}$ mice by gene targeting (Supplementary Figure 2a-d). Eightweek-old $R n f 186^{-1-}$ mice reared in a specific pathogen-free facility did not show any obvious histological abnormality in the large intestine (Supplementary Figure 3a). Although RNF186 has been shown to localize at ER, ${ }^{17}$ the Rnf186 deficiency did not induce an apparent morphological change of ER in the colonic epithelia (Supplementary Figure 3b). Development of Paneth cells and goblet cells of ileum was normally observed in $R n f 186^{-/-}$mice (Supplementary
Figure 4a-c). Histological sections stained with hematoxylin and eosin did not show any intensive intestinal inflammation in 1-year-old $R n f 186^{-1-}$ mice compared with age-matched $R n f 186^{+/+}$mice (Supplementary Figure 5a). In addition, expression of inflammatory cytokines, such as $I l 1 b$, Il6, Il12p40, Il17a, Ifng, and Tnfa, was similar in 1-year-old Rnf186 $6^{+/+}$and Rnf186 ${ }^{-1-}$ colonic tissues (Supplementary Figure 5b). Moreover, percentages and numbers of IFN- $\gamma-$ and IL-17- expressing CD4 ${ }^{+} \mathrm{T}$ cells were not altered in the colonic lamina propria of 1-year-old $R n f 186^{-1-}$ mice compared with age-matched $R n f 186^{+/+}$mice (Supplementary Figure 6). These data suggest that $R n f 186^{-1-}$ mice grew healthily without any inflammatory changes in their large intestines. To evaluate the gastrointestinal barrier function, we examined gastrointestinal tract permeability in 8-week-old and 1-yearold $R n f 186^{+/+}$and $R n f 186^{-1-}$ mice. The permeability for fluorescein isothiocyanate (FITC)-dextran $(4,400 \mathrm{Da})$ was higher in Rnf186 ${ }^{-/-}$mice than in $R n f 186^{+/+}$mice (Figure 1c; Supplementary Figure 7a). In addition, the paracellular flux determined by measuring the paracellular permeability across the colon epithelium was enhanced by the Rnf186 deficiency for the small organic solute lucifer yellow $(457 \mathrm{Da})$ (Figure 1d; Supplementary Figure $7 \mathbf{b}$ ). We next measured the electrophysiology across the large intestinal mucosa in $R n f 186^{+/+}$and Rnf186 ${ }^{-1-}$ mice in an Ussing chamber system. The permeability of inorganic ions in the large intestine was similar in $\mathrm{Rnfl}_{186^{+/+}}$and $\mathrm{Rnfl} 186^{-/-}$mice (Figure 1e and f; Supplementary Figure $7 \mathrm{c}$ and d). Thus, the permeability of small organic solutes, such as dextran $(4,400 \mathrm{Da})$ and lucifer yellow $(457 \mathrm{Da})$, but not inorganic ions, was increased in the intestines of $R n f 186^{-1-}$ mice.

\section{Regulation of occludin expression by RNF186}

Tight junction molecules control gastrointestinal tract permeability. ${ }^{20,21}$ Because $R n f 186^{-1-}$ mice showed increased permeability in their colon, we examined messenger RNA (mRNA) expression of genes encoding various tight junction proteins including Ocln, Cldn2, Cldn3, Cldn4, Cldn7, Cldn15, and Tjp1 in colonic epithelial cells (Figure 2a; Supplementary Figure 8). Among these genes, expression of $\mathrm{Ocln}$ (encoding occludin) was substantially increased in $R n f 186^{-1-}$ mice. Occludin protein expression was also increased in $R n f 186^{-1-}$ colonic epithelia (Figure 2b). As a component of tight junction molecules, occludin was exclusively expressed at the contact junction of closely-attached colonic epithelial cells of $R n f 186^{+1+}$ mice in a whole-mount immunohistochemical analysis (Figure 2c). The occludin expression was altered in $R n f 186^{-1-}$ mice. The higher magnification of the immuno-stained sections showed selective occludin expression at the apical side of the colonic epithelia of Rnf186 ${ }^{+/+}$mice (Figure 2d). However, occludin was broadly expressed in the cytoplasm of colonic epithelia of $R n f 186^{-1-}$ mice. Thus, Rnf186 ${ }^{-1-}$ mice showed an altered expression pattern of occludin in colonic epithelia.

RNF186 acts as an E3 ubiquitin ligase in the ER membrane. ${ }^{17}$ In addition, occludin is a target of the E3 ligase Itch for its 

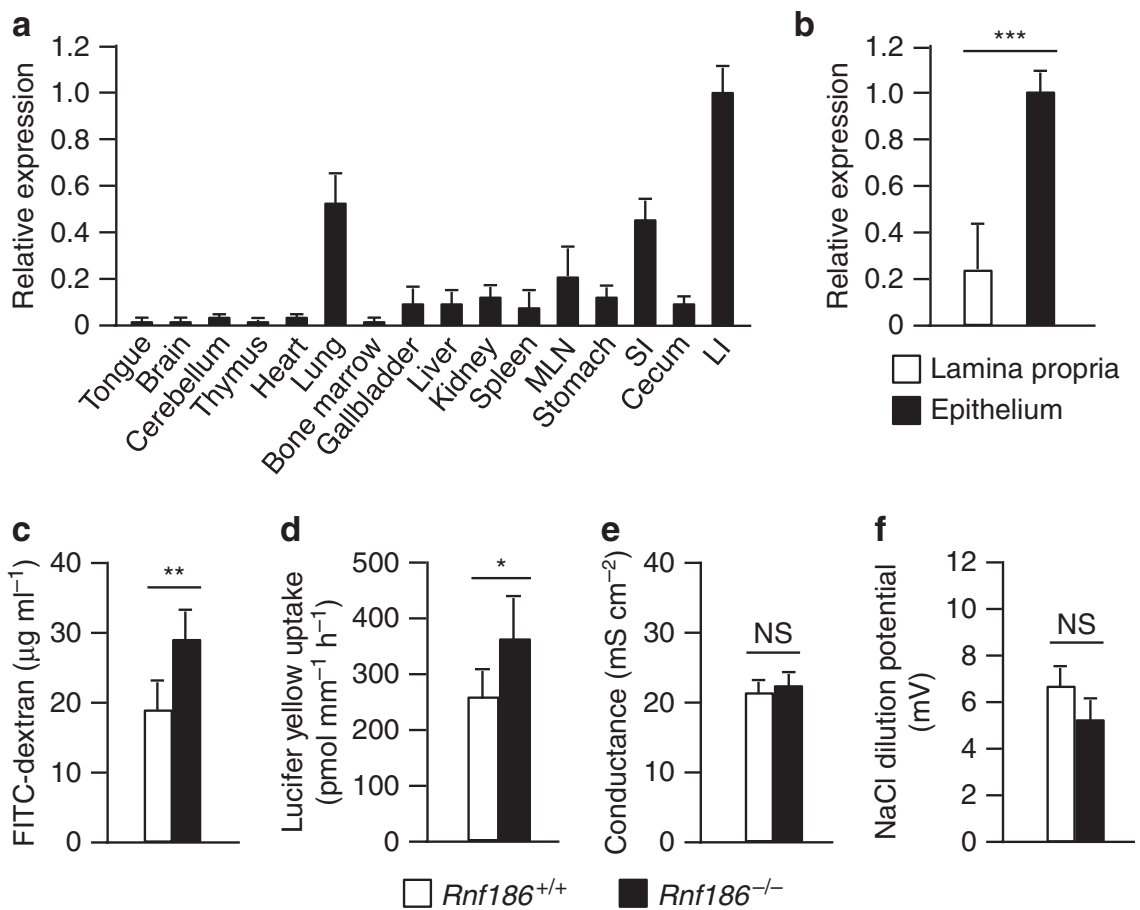

Figure 1 High colonic permeability of Rnf186 ${ }^{-l-}$ mice. (a) Quantitative RT-PCR analysis of Rnf186 mRNA expression in various organs $(n=3$ per group). The values were normalized to Gapdh expression. Data are representative of three independent experiments. (b) Quantitative RT-PCR analysis of Rnf186 mRNA expression in the lamina propria and epithelium of the large intestine ( $n=4$ per group). The values were normalized to Gapdh expression. Data are representative of two independent experiments. Data are shown as the mean \pm s.d. ${ }^{* \star \star} P<0.001$ by Student's $t$ test. (c) Epithelial barrier function against FITC-dextran in 8-week-old $R n f 186^{+/+}$and $R n f 186^{-/-}$mice $\left(n=5\right.$ per group). Data are shown as the mean $\pm \mathrm{s}$.d. ${ }^{* \star} P<0.01$ by Student's $t$ test. (d) Rate of lucifer yellow uptake into 8-week-old Rnf186 ${ }^{+1+}(n=5)$ and $R n f 186^{-1-}(n=9)$ colons. Data are shown as the mean \pm s.d. ${ }^{\star} P<0.05$ by Student's $t$ test. (e) Statistical analyses of the ionic conductances across the large intestines of 8 -week-old $R n f 186^{+1+}$ and $R n f 186^{-1-}$ mice ( $n=5$ per group). Data are shown as the mean \pm s.d. NS: not significant by Student's $t$ test. (f) Electrophysiological analyses of the NaCl dilution potential in the large intestines of 8-week-old Rnf186 $6^{+1+}$ and $R n f 186^{-1-}$ mice ( $n=5$ per group). Data are shown as the mean \pm s.d. NS: not significant by Student's $t$ test. LI, large intestine; MLN, mesenteric lymph node; SI, small intestine.

protein turnover. ${ }^{22}$ Therefore, we examined whether RNF186 catalyzes polyubiquitination of occludin. RNF186 was colocalized with occludin at ER in Caco-2 human colonic epithelial cells (Figure 2e). We next examined whether occludin was a substrate of RNF186. Flag-tagged human RNF186 (Flag-RNF186 ${ }^{\mathrm{WT}}$ ), Myc-tagged occludin (Myc-Occludin), and HA-tagged ubiquitin (HA-Ub) were co-expressed in HEK293T cells, followed by analysis of the ubiquitination levels of occludin (Figure 2f). Flag$\mathrm{RNF} 186^{\mathrm{WT}}$ induced extensive polyubiquitination of occludin compared with a mutant RNF186 (Flag-RNF186 ${ }^{\mathrm{H} 60 \mathrm{~W}}$ ) in which histidine at position 60 of the RING finger domain was substituted with tryptophan, leading to defective E3 ligase activity. ${ }^{23}$

We next analyzed whether RNF186 catalyzes K48-linked or K63-linked ubiquitination of occludin. Flag-RNF186 $6^{\mathrm{WT}}$ mediated polyubiquitination of occludin was detected by anti-K48 ubiquitin antibody, but not by anti-K63 ubiquitin antibody (Figure 2g; Supplementary Figure 9). Furthermore, polyubiquitination of occludin was not induced when HA-tagged K48-linked ubiquitin mutant $\left(\mathrm{HA}-\mathrm{Ub}^{\mathrm{K} 48 \mathrm{R}}\right)^{24}$ was introduced (Figure 2h). These data suggest that RNF186 is an E3 ubiquitin ligase controlling the homeostasis of occludin.

\section{Altered protein expression pattern in $R n f 186^{-I-}$ colonic epithelia}

Because RNF186 was found to mediate K48-linked polyubiquitination, which contributes to regulation of protein turnover through degradation by targeting to the proteasome, ${ }^{12}$ we compared the expression of colonic epithelial proteins in $R n f 186^{+/+}$and $R n f 186^{-1-}$ mice by liquid chromatographytandem mass spectrometry (LC-MS/MS). Quantitative proteomic analysis using normalized total spectra showed that 52 proteins were found to be significantly different between $R n f 186^{+/+}$and $R n f 186^{-1-}$ colonic epithelial cells (Figure 3a; Table 1; Supplementary Table 1). A dataset of 52 proteins was used for the canonical pathway analysis in ingenuity pathway analysis (IPA), which identifies signaling pathways based on Kyoto Encyclopedia of Genes and Genomes canonical pathway analysis (http://www.genome.jp/kegg; Supplementary Figure 10). Among down-regulated proteins, several pathways were affected and the pathway of LPS/IL1-mediated inhibitor of RXR functions was the most significant. In contrast, the protein ubiquitin pathway was the sole pathway that was significantly altered. We also performed functional analysis in IPA, which identifies biological relevance on the basis of manually curated literature findings. In diseases and disorders category, 
a

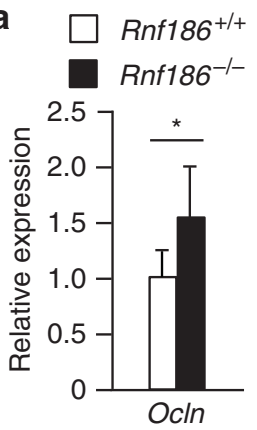

C

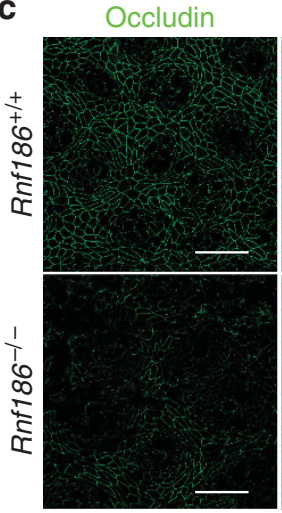

Occludin+DAPI

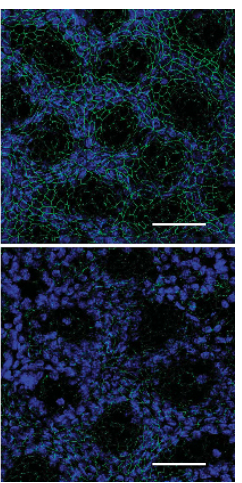

f

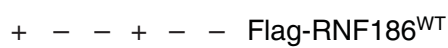

-+--+- Flag-RNF186 ${ }^{\mathrm{H} 60 \mathrm{~W}}$

-++++- Myc-Occludin

(kD)

$++++++\mathrm{HA}-\mathrm{Ub}$

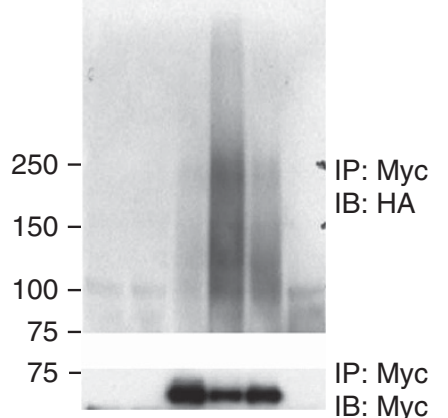

Rnf186 $6^{+/+}$

Rnf186-/-

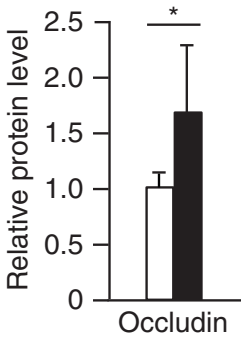

d

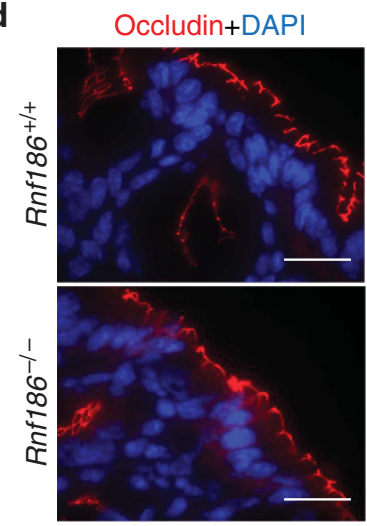

9

$$
\begin{aligned}
& +\quad-\text { Flag-RNF186 WT } \\
& -\quad+\text { Flag-RNF186 } \\
& +\quad+\text { Myc-Oc } \\
& +\quad+\text { HA-Ub }
\end{aligned}
$$

(kD)

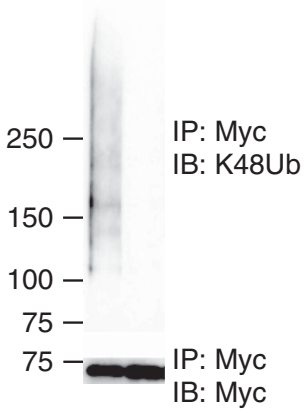

e

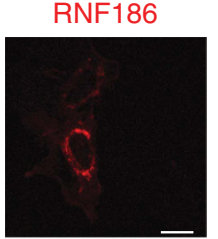

Occludin

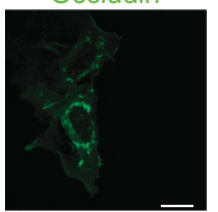

KDEL

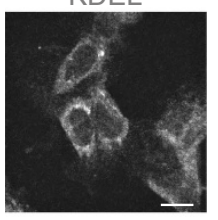

Merge

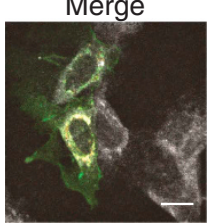

h

$$
++ \text { Flag-RNF186 }{ }^{\text {WT }}
$$

++ Myc-Occludin

$+-\mathrm{HA}-\mathrm{Ub}$

(kD) $-+\mathrm{HA}-\mathrm{Ub}^{\mathrm{K} 48 \mathrm{R}}$
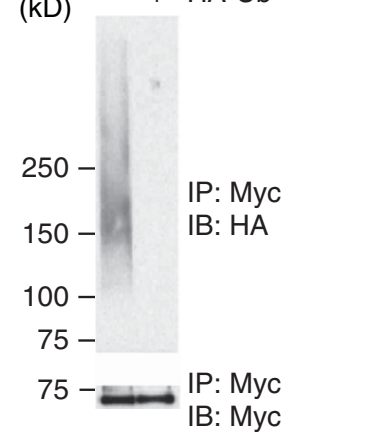

Figure 2 Polyubiquitination of occludin by RNF186. (a) Quantitative RT-PCR analysis of Ocln mRNA expression in colonic epithelial cells of 8-week-old $R n f 186^{+1+}$ and $R n f 186^{-1-}$ mice ( $n=6$ per group). The values were normalized to Gapdh expression. Data are representative of two independent experiments. Data are shown as the mean \pm s.d. ${ }^{*} P<0.05$ by Student's $t$ test. (b) Immunoblot analysis of occludin in whole-cell lysates of colonic epithelial cells from 8-week-old Rnf186 ${ }^{+1+}$ and Rnf186 ${ }^{-1-}$ mice. Bottom, immunoblot analysis of actin (loading control). A representative blot of two independent experiments is shown. The right panel shows the densitometry of western blot signals with statistical evaluation. Data are shown as the mean $\pm \mathrm{s}$. $\mathrm{d}$. of four mice analyzed. ${ }^{*} P<0.05$ by Student's $t$ test. (c) Immunofluorescence staining of whole-mount colon samples with an anti-occludin antibody (green) and DAPI (blue). Original magnification, $\times 40$. Scale bar, $50 \mu \mathrm{m}$. Data are representative of three independent experiments. (d) Immunofluorescence staining of frozen colon sections with an anti-occludin antibody (red) with DAPI staining (blue). Original magnification, $\times 100$. Scale bar, $25 \mu \mathrm{m}$. Data are representative of two independent experiments. (e) Expression of mCherry-RNF186 (red), GFP-occludin (green), and KDEL (white) in Caco-2 cells. Scale bar, $10 \mu \mathrm{m}$. Data are representative of three independent experiments. (f-h) Immunoblot analysis of anti-Myc immunoprecipitates from lysates of HEK293 cells co-transfected with the indicated expression vectors using anti-HA or anti-Myc antibodies (f,h), and anti-K48 ubiquitin or anti-Myc antibodies (g). Data are representative of more than three independent experiments. DAPI, 4',6-diamidino-2-phenylindole.

52 proteins were associated with gastrointestinal diseases (Figure 3b). These findings raised the possibility that RNF186 regulates expression level of these proteins, and the altered expression patterns of these proteins are associated with the pathogenesis of intestinal inflammation. To determine direct targets of RNF186, we examined whether RNF186 interacts with these proteins, particularly focusing on proteins, which expression was increased in $R n f 186^{-/-}$ 


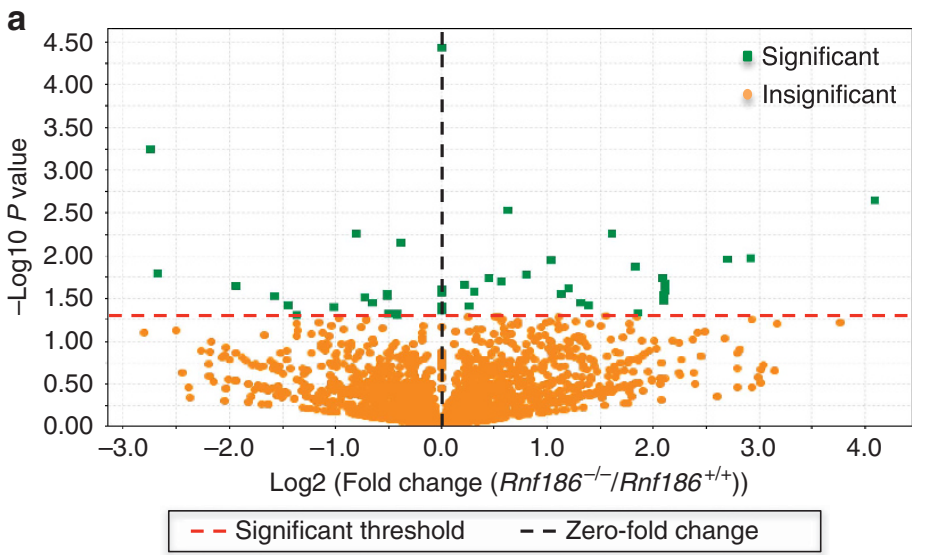

b
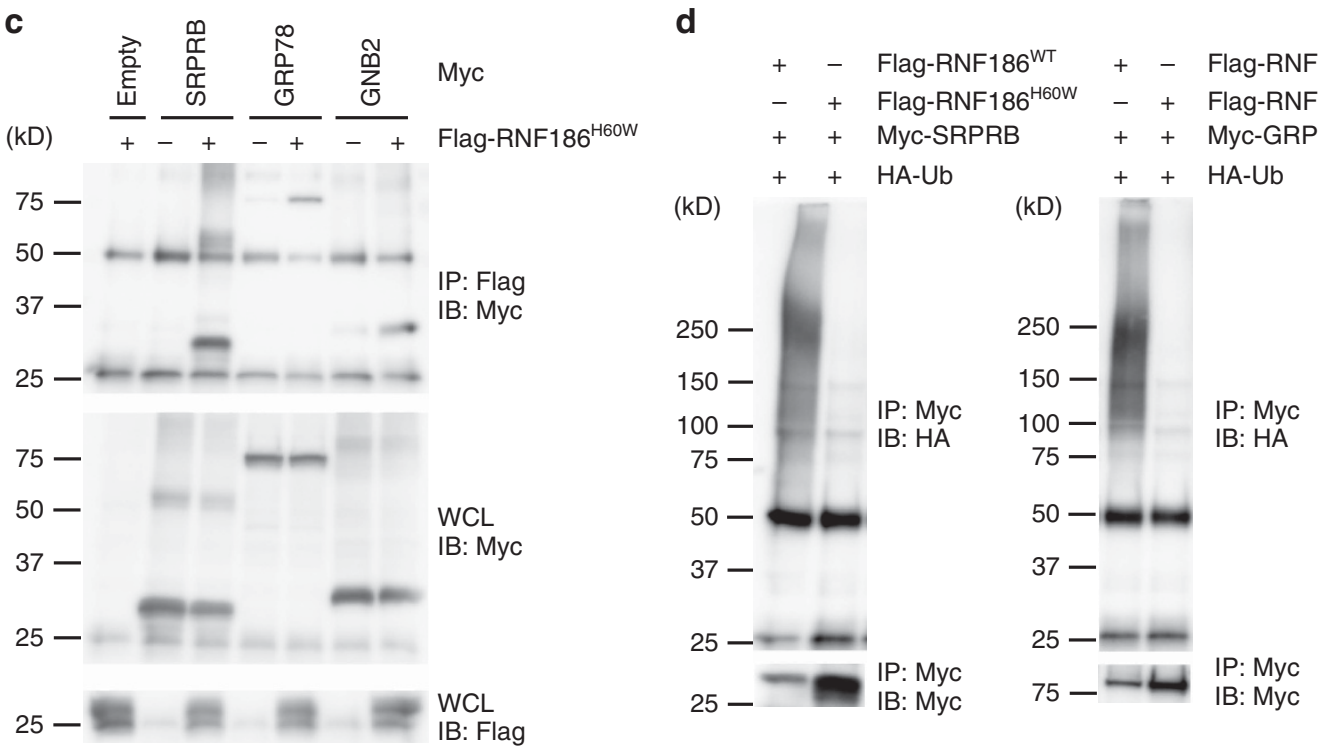

\begin{tabular}{cc}
\hline Diseases and disorders & $\boldsymbol{P}$ value \\
\hline Gastrointestinal disease & $1.48 \mathrm{E}-02-4.27 \mathrm{E}-04$ \\
\hline Hepatic system disease & $9.91 \mathrm{E}-03-4.27 \mathrm{E}-04$ \\
\hline Hereditary disorder & $3.67 \mathrm{E}-02-4.27 \mathrm{E}-04$ \\
\hline Auditory disease & $1.24 \mathrm{E}-02-2.49 \mathrm{E}-03$ \\
\hline Developmental disorder & $3.67 \mathrm{E}-02-2.49 \mathrm{E}-03$ \\
\hline
\end{tabular}

Figure 3 Identification of RNF186 substrates. (a) Volcano plot displaying the averaged log 2 fold change for LC-MS/MS proteomics data obtained from colonic epithelial cells of 8 -week-old $R n f 186^{+1+}$ and $R n f 186^{-1-}$ mice $(n=4$ per group) ( $x$-axis) and their adjusted negative logarithm base 10 of the $P$ value ( $y$-axis). Green squares indicate proteins with statistically difference $(P<0.05$; moderated $t$ test, adjusted by the Benjamini-Hochberg method $(\mathrm{BH})$ ), whereas orange squares indicate proteins with insignificant difference. (b) Top five diseases and disorders, with which 52 significant proteins identified in (a) were associated. $P$ values are calculated by IPA. (c) Immunoblot analysis of anti-Flag immunoprecipitates (upper) and whole-cell lysates (middle and bottom) of HEK293 cells co-transfected with the indicated expression vectors using anti-Myc or anti-Flag antibodies. Data are representative of three independent experiments. (d) Immunoblot analysis of anti-Myc immunoprecipitates from lysates of HEK293 cells co-transfected with the indicated expression vectors using anti-HA or anti-Myc antibodies. Data are representative of two independent experiments. IPA, ingenuity pathway analysis; LC-MS/MS, liquid chromatography-tandem mass spectrometry.

colonic epithelial cells. Among six proteins analyzed, signal recognition particle receptor, B subunit (SRPRB), glucoseregulated protein, $78 \mathrm{kDa}$ (GRP78) and guanine nucleotide binding protein, beta polypeptide 2 (GNB2) interacted with $\mathrm{RNF} 186^{\mathrm{H} 60 \mathrm{~W}}$ (Figure 3c; Supplementary Figure 11). As is the case in occludin (Figure 2f), Flag-RNF186 ${ }^{\mathrm{WT}}$, but not Flag-RNF186 ${ }^{\mathrm{H} 60 \mathrm{~W}}$, induced polyubiquitination of SRPRB, GRP78, and GNB2 (Figure 3d). In addition, in cells expressing Flag-RNF186 ${ }^{\mathrm{WT}}$, expression of SRPRB, GRP78, and GNB2 was severely decreased compared to cells expressing Flag-RNF186 ${ }^{\mathrm{H} 60 \mathrm{~W}}$ (Figure 3d). These findings indicate that RNF186 regulates expression of several proteins, such as SRPRB, GRP78, and GNB2 through induction of polyubiquitination.

\section{Deterioration of DSS-induced colitis in $\mathrm{Rnf186}^{-{ }^{-}}$mice}

RNF186 was found to regulate the homeostasis of several colonic epithelial proteins including occludin, SRPRB, GRP78, and GNB2. Therefore, we next analyzed whether the dysregulated protein homeostasis in colonic epithelial cells of $R n f 186^{-1-}$ mice leads to an increased risk of intestinal inflammation. To this end, DSS was orally administrated to $R n f 186^{+/+}$and $R n f 186^{-1-}$ mice. Rnf186 ${ }^{-1-}$ mice showed severe weight loss and a decreased survival rate compared with $R n f 186^{+/+}$mice (Figure 4a,b). Histological sections stained with hematoxylin and eosin showed intensive intestinal inflammation in $R n f 186^{-1-}$ mice compared with $R n f 186^{+/+}$ mice after 4 and 8 days of DSS administration (Figure 4c). Protein expression of SRPRB, GRP78, and GNB2 was enhanced 
Table 1 List of significant proteins in $R_{n f 186^{-I-}}$ mice

\begin{tabular}{|c|c|c|c|c|c|c|c|c|c|c|}
\hline Identified proteins & Accession number & WT1 & WT2 & WT3 & WT4 & KO1 & KO2 & KO3 & KO4 & $\boldsymbol{P}$ value \\
\hline Stt3a & IPI00109108 & 8.4371 & 10.794 & 7.1096 & 10.165 & 12.649 & 14.926 & 13.5 & 15.036 & 0.0029 \\
\hline 2310003C23Rik & IPI00110487 & 1.298 & 1.1362 & 0 & 0 & 1.6499 & 1.7223 & 3.3749 & 3.7589 & 0.021 \\
\hline Mrps28 & IPI00110672 & 0 & 0 & 0 & 0 & 2.1998 & 2.8705 & 3.3749 & 0 & 0.03 \\
\hline Acsl1 & IPI00112549 (+ 1) & 4.543 & 8.5218 & 7.1096 & 3.3882 & 9.8992 & 13.204 & 10.125 & 15.036 & 0.011 \\
\hline Trim2 & IPI00113430 (+3) & 0 & 0 & 0 & 0 & 1.6499 & 0 & 3.3749 & 3.7589 & 0.044 \\
\hline Chga & IPI00113703 & 2.596 & 5.1131 & 7.1096 & 6.7764 & 2.7498 & 2.8705 & 0 & 0 & 0.023 \\
\hline Anxa13 & IPI00115275 & 5.841 & 5.1131 & 7.1096 & 6.7764 & 0 & 5.7409 & 3.3749 & 0 & 0.037 \\
\hline Reg3b & IPI00116548 & 0 & 0 & 0 & 0 & 6.0495 & 4.0187 & 0 & 3.7589 & 0.034 \\
\hline Cdk5rap3 & IPI00117025 & 0.64901 & 0 & 0 & 0 & 1.6499 & 2.2964 & 3.3749 & 3.7589 & 0.0022 \\
\hline Ctsh & IPI00118987 & 0 & 0 & 0 & 0 & 3.2997 & 4.0187 & 3.3749 & 0 & 0.026 \\
\hline Aldoc & IPI00119458 & 0 & 0 & 0 & 0 & 3.5548 & 3.3882 & 2.2725 & 3.245 & $<0.00010$ \\
\hline Ndufa9 & IPI00120212 & 29.205 & 22.157 & 31.993 & 30.494 & 22.548 & 18.945 & 23.624 & 15.036 & 0.028 \\
\hline Nup50 & IPI00120572 & 0 & 3.4087 & 3.5548 & 3.3882 & 4.3996 & 5.1668 & 6.7498 & 7.5178 & 0.024 \\
\hline Trim25 & IPI00122289 (+5) & 1.947 & 2.8406 & 3.5548 & 3.3882 & 1.6499 & 2.2964 & 0 & 0 & 0.03 \\
\hline Rpl9 & IPI00122413 (+ 1) & 9.0861 & 8.5218 & 10.664 & 10.165 & 7.1494 & 8.0373 & 6.7498 & 7.5178 & 0.0071 \\
\hline Rbbp4 & IPI00122696 (+1) & 0 & 4.545 & 3.5548 & 3.3882 & 0 & 0 & 0 & 0 & 0.027 \\
\hline Pafah2 & IPI00123270 (+ 1) & 5.192 & 0 & 0 & 0 & 4.9496 & 6.8891 & 6.7498 & 3.7589 & 0.029 \\
\hline 2610301G19Rik & IPI00123624 & 6.4901 & 8.5218 & 3.5548 & 0 & 9.8992 & 9.1855 & 10.125 & 11.277 & 0.028 \\
\hline Clcn2 & IPI00127238 & 0 & 7.3856 & 3.5548 & 6.7764 & 9.8992 & 12.63 & 6.7498 & 15.036 & 0.036 \\
\hline Ggh & IPI00129243 (+ 1) & 11.682 & 13.635 & 17.774 & 16.941 & 10.999 & 12.63 & 10.125 & 11.277 & 0.048 \\
\hline Eif3a & IPI00129276 & 9.0861 & 13.067 & 10.664 & 13.553 & 7.1494 & 5.1668 & 6.7498 & 7.5178 & 0.0055 \\
\hline Sult1b1 & IPI00131365 (+ 1) & 8.4371 & 7.9537 & 7.1096 & 13.553 & 7.6994 & 3.4446 & 3.3749 & 3.7589 & 0.04 \\
\hline Cops3 & IPI00131870 & 0 & 0 & 0 & 0 & 1.6499 & 0 & 3.3749 & 3.7589 & 0.044 \\
\hline Fam136a & IPI00133411 & 3.245 & 2.2725 & 0 & 0 & 3.2997 & 4.0187 & 3.3749 & 3.7589 & 0.037 \\
\hline Psmc1 & IPI00133428 & 1.947 & 5.6812 & 0 & 0 & 5.4996 & 7.4632 & 6.7498 & 7.5178 & 0.014 \\
\hline Mut & IPI00133553 & 18.172 & 13.635 & 17.774 & 20.329 & 13.749 & 14.352 & 6.7498 & 7.5178 & 0.031 \\
\hline Tppp3 & IPI00133557 & 1.947 & 2.2725 & 3.5548 & 0 & 0 & 0 & 0 & 0 & 0.038 \\
\hline Slc25a13 & IPI00135651 (+2) & 27.907 & 22.725 & 28.439 & 23.717 & 21.998 & 23.538 & 13.5 & 15.036 & 0.047 \\
\hline Mrpl24 & IPI00162769 & 0 & 0 & 0 & 0 & 2.7498 & 1.7223 & 3.3749 & 0 & 0.037 \\
\hline Gnb2 & IPI00162780 (+ 1) & 18.172 & 15.339 & 14.219 & 16.941 & 18.149 & 18.945 & 20.249 & 22.554 & 0.026 \\
\hline Glt25d1 & IPI00169870 & 0 & 0 & 0 & 0 & 2.1998 & 0 & 3.3749 & 3.7589 & 0.033 \\
\hline Cd9 & IPI00221921 & 11.033 & 7.3856 & 14.219 & 10.165 & 6.0495 & 6.8891 & 6.7498 & 7.5178 & 0.035 \\
\hline Mrps35 & IPI00222538 (+ 1) & 0 & 0 & 0 & 3.3882 & 3.2997 & 4.0187 & 3.3749 & 3.7589 & 0.019 \\
\hline Tprn & IPI00223286 & 0 & 0 & 0 & 0 & 2.1998 & 2.2964 & 3.3749 & 0 & 0.032 \\
\hline Dguok & IPI00224955 & 1.298 & 2.2725 & 3.5548 & 3.3882 & 1.6499 & 0 & 0 & 0 & 0.016 \\
\hline Epb4.112 & IPI00309481 (+ 1) & 0 & 2.2725 & 0 & 0 & 2.7498 & 4.0187 & 6.7498 & 3.7589 & 0.011 \\
\hline Ccdc47 & IPI00310518 & 4.543 & 5.6812 & 3.5548 & 6.7764 & 7.6994 & 8.6114 & 6.7498 & 7.5178 & 0.02 \\
\hline Stx17 & IPI00316431 & 0 & 3.9768 & 0 & 0 & 4.3996 & 2.8705 & 3.3749 & 3.7589 & 0.047 \\
\hline Grp78 & IPI00319992 & 114.87 & 111.35 & 110.2 & 125.36 & 122.09 & 133.19 & 135 & 146.6 & 0.022 \\
\hline Fmo2 & IPI00322245 & 4.543 & 3.4087 & 3.5548 & 3.3882 & 1.6499 & 0.57409 & 0 & 0 & 0.00056 \\
\hline Kdelc2 & IPI00344686 & 0 & 1.7044 & 0 & 0 & 1.6499 & 2.2964 & 3.3749 & 3.7589 & 0.011 \\
\hline Psmd4 & IPI00381291 & 0 & 2.8406 & 0 & 0 & 1.6499 & 3.4446 & 3.3749 & 3.7589 & 0.034 \\
\hline Mgat4c & IPI00387409 & 0 & 4.545 & 3.5548 & 3.3882 & 9.8992 & 10.908 & 6.7498 & 7.5178 & 0.0055 \\
\hline Set & IPI00410883 & 3.894 & 3.4087 & 3.5548 & 0 & 0 & 0 & 0 & 0 & 0.025 \\
\hline Heatr1 & IPI00411022 & 0 & 3.9768 & 0 & 0 & 4.3996 & 5.7409 & 3.3749 & 3.7589 & 0.025 \\
\hline Ugt1a7c & IPI00417181 & 47.377 & 34.655 & 42.658 & 47.434 & 34.097 & 37.316 & 26.999 & 22.554 & 0.03 \\
\hline
\end{tabular}


Table 1 (Continued)

\begin{tabular}{llrrrrrrrrr}
\hline Identified proteins & Accession number & \multicolumn{1}{c}{ WT1 } & WT2 & WT3 & WT4 & KO1 & KO2 & KO3 & KO4 & $\boldsymbol{P}$ value \\
\hline Mat2b & IPI00468802 (+1) & 3.894 & 1.7044 & 3.5548 & 0 & 0 & 0 & 0 & 0 & 0.044 \\
Duox2 & IPI00606008 (+1) & 10.384 & 7.3856 & 7.1096 & 16.941 & 19.248 & 17.797 & 16.875 & 18.795 & 0.017 \\
Cog7 & IPI00623090 & 0 & 0 & 0 & 0 & 3.2997 & 0 & 3.3749 & 3.7589 & 0.025 \\
Comt1 & IPI00759876 & 7.1391 & 5.6812 & 7.1096 & 6.7764 & 5.4996 & 6.315 & 3.3749 & 3.7589 & 0.047 \\
Lima1 & IPI00759925 & 27.907 & 27.838 & 24.884 & 23.717 & 30.248 & 33.872 & 26.999 & 33.83 & 0.039 \\
Srprb & IPI00987951 & 13.629 & 18.18 & 17.774 & 16.941 & 21.998 & 26.408 & 23.624 & 18.795 & 0.019 \\
\hline
\end{tabular}

The colonic epithelial proteins in 8-week-old Rnf186 ${ }^{+/+}(\mathrm{WT})$ and $R n f 186^{-\prime-}(\mathrm{KO})$ mice $(n=4$ per group) were analyzed by LC-MS/MS. List of proteins, which were statistically significant by quantitative proteomic analysis, is shown. The normalized total spectra, which were analyzed with Scaffold (ver. 4.4.5), are represented. $P$ value by moderated $t$ test, adjusted by the Benjamini-Hochberg method $(\mathrm{BH})$. Red letters show upregulated proteins and blue letters show down-regulated proteins in Rnf186 ${ }^{-1-}$ mice.

in the colonic epithelial cells of DSS-treated $R n f 186^{-/-}$mice compared with those of DSS-treated $R n f 186^{+/+}$mice (Figure 4d). Thus, Rnf186 ${ }^{-1-}$ mice were highly susceptible to DSS-induced intestinal inflammation with the increased expression of RNF186 target proteins.

In DSS-mediated colitis, induction of the UPR has been demonstrated in colonic epithelial cells. ${ }^{25,26}$ In addition, GRP78, which was increased in $R n f 186^{-1-}$ colonic epithelia (Figure 4d), was integral to UPR ${ }^{13}$ Therefore, we analyzed mRNA expression of another UPR-related gene Ddit3, encoding CHOP. ${ }^{27,28}$ Expression of Ddit3 was dramatically increased in colonic epithelial cells of $R n f 186^{-1-}$ mice after DSS treatment (Supplementary Figure 12). Moreover, in the colonic epithelial cells of DSS-treated $R n f 186^{-1-}$ mice, protein expression of CHOP and spliced XBP1 (sXBP1) was markedly augmented (Figure 5a). Thus, the deficiency of Rnf186 enhanced ER stress in colonic epithelial cells during DSS administration.

To determine the association between intestinal inflammation and cell death of colonic epithelial cells in $R n f 186^{+/+}$and $R n f 186^{-1-}$ mice, we examined the number of TUNEL- and active caspase-3-positive cells among colonic epithelial cells of $R n f 186^{+1+}$ and $R n f 186^{-1-}$ mice before and after 4 days of DSS administration. Marked increases in the numbers of TUNEL- and active caspase-3-positive cells were detected in the colonic epithelial cells of DSS-treated $R n f 186^{-1-}$ mice compared with $\mathrm{Rnfl} 186^{+/+}$mice (Figure $\mathbf{5 b}, \mathbf{c}$ ). These findings indicate that the deficiency of Rnf186 leads to an increase in apoptosis of colonic epithelial cells together with enhanced ER stress. We also introduced another model of intestinal inflammation using oxazolone, which is reported to induce UC-like histologic changes in the large intestine $^{29}$ (Supplementary Figure 13a,b). Oxazolone-treated Rnf186 ${ }^{-1-}$ mice suffered from the more severe intestinal inflammation than $R n f 186^{+1+}$ mice, as evidenced by profound pathology and weight loss. Thus, $R n f 186^{-1-}$ mice were highly sensitive to intestinal inflammation induced by foreign stress.

To test the effects of ER stress on colonic epithelial integrity in $R n f 186^{-1-}$ mice more directly, we intraperitoneally challenged mice with an ER stress inducer, tunicamycin. Whereas only one of $13 \mathrm{Rnf186^{+/+ }}$ mice died after tunicamycin challenge, 13 of 18 Rnf $186^{-1-}$ mice succumbed within $96 \mathrm{~h}$ (Figure 6a). TUNEL staining of colonic tissues showed an increased number of dead cells in $R n f 186^{-/-}$mice compared with that in $R n f 186^{+/+}$mice (Figure $6 \mathbf{b}$ ). Thus, RNF186 is required for protection of colonic epithelial cells from tunicamycin-induced ER stress.

Impaired function of the UC-associated variant of RNF186 We next analyzed whether substitution of alanine with threonine at the 64th position (A64T), which is found in UC patients, ${ }^{9}$ was associated with the increased risk of intestinal inflammation. The human RNF186 A64T mutant (Flag-RNF186 ${ }^{\mathrm{A} 64 \mathrm{~T}}$ ) induced less polyubiquitination of occludin than Flag-RNF186 ${ }^{\mathrm{WT}}$ (Figure 7a). Thus, the A64T mutation leads to dysfunction of the RNF186 protein.

Next, we generated A64T knock-in mice (Rnf186 $\left.{ }^{\mathrm{A} 117 \mathrm{~T} / \mathrm{A} 117 \mathrm{~T}}\right)$ in which alanine at the 117th position (corresponding to the 64th position in human RNF186) was substituted with threonine using the CRISPR/Cas9 system $^{30}$ (Supplementary Figure 14a,b). The uptake of lucifer yellow in colonic epithelial cell layers was enhanced in $R n f 186^{\mathrm{A} 117 \mathrm{~T} / \mathrm{A} 117 \mathrm{~T}}$ mice (Figure 7b). Rnf186 ${ }^{\mathrm{A} 117 \mathrm{~T} / \mathrm{A} 117 \mathrm{~T}}$ mice showed higher sensitivity to tunicamycin-induced ER stress than $R n f 186^{\mathrm{wt} / \mathrm{wt}}$ mice (Figure 7c). In addition, Rnf186 ${ }^{\mathrm{A} 117 \mathrm{~T} / \mathrm{A} 117 \mathrm{~T}}$ mice showed more severe weight loss and a decreased survival rate after DSS administration compared with $R n f 186^{\mathrm{wt} / \mathrm{wt}}$ mice (Figure 7d,e). Histological analyses showed intensive intestinal inflammation with increased numbers of TUNEL-positive cells in $R n f 186^{\mathrm{A} 117 \mathrm{~T} / \mathrm{A} 117 \mathrm{~T}}$ mice compared with $\mathrm{R} n f 186^{\mathrm{wt} / \mathrm{wt}}$ mice after DSS administration (Figure 7f,g). Furthermore, expression of GRP78, CHOP, and sXBP1 in colonic epithelial cells was markedly increased in DSS-treated Rnf186 ${ }^{\mathrm{A} 117 \mathrm{~T} / \mathrm{A} 117 \mathrm{~T}}$ mice (Figure 7h). These findings indicate that the A117T mutation in mice (A64T in humans) correlates with an increased risk for stress-induced intestinal inflammation.

\section{DISCUSSION}

In the present study, we analyzed the functional role of the UC-associated gene Rnf186 in mice, ${ }^{6-9}$ and demonstrated that RNF186 is responsible for the maintenance of intestinal homeostasis in colonic epithelial cells.

RNF186 is an E3 ligase that is highly expressed in colonic epithelial cells, which regulates the protein homeostasis (proteostasis) $^{31,32}$ of its substrates through induction of K48-linked polyubiquitination. Among the substrates 


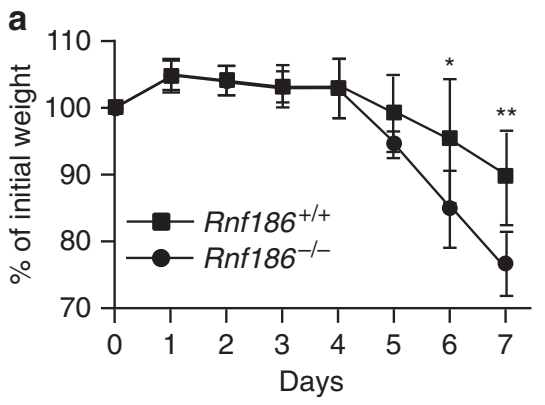

C

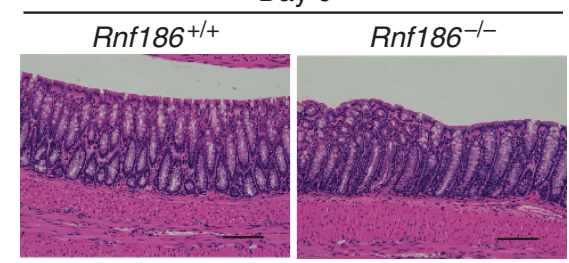

Day 4

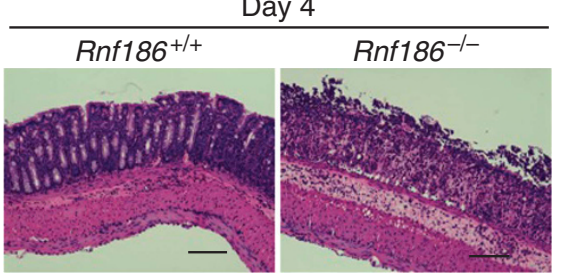

Day 8

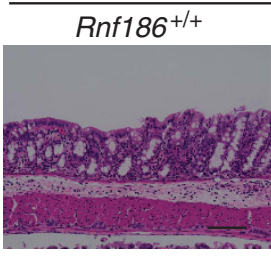

Rnf186 ${ }^{-/-}$

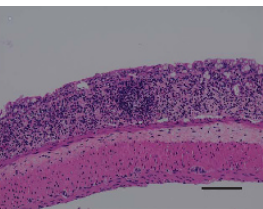

b

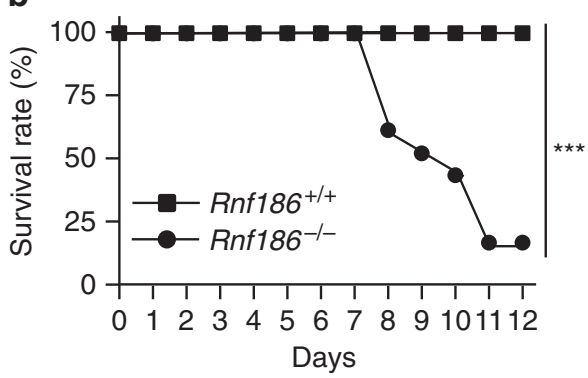

O Rnf186 $+/+$

- Rnf186 ${ }^{-/}$

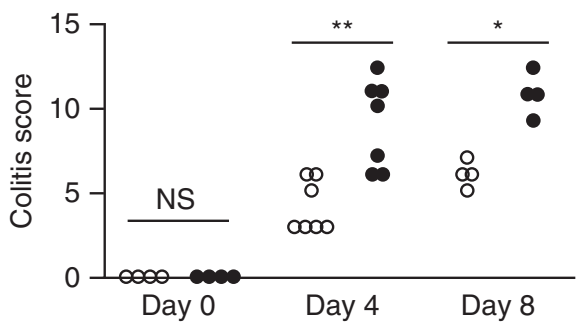

d
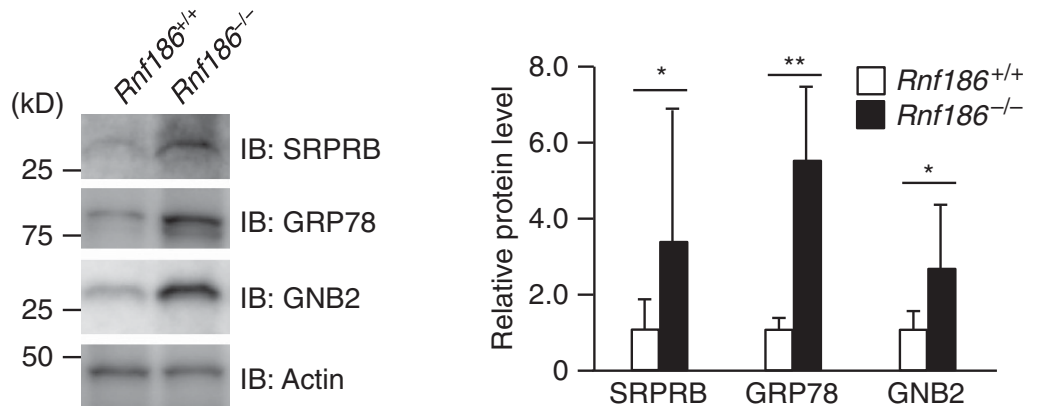

Figure 4 High sensitivity to DSS-induced intestinal inflammation in Rnf186 ${ }^{-/-}$mice. (a-c) Eight-week-old $R n f 186^{+/+}(n=10)$ and $R n f 186^{-1-}$ mice $(n=9)$ were orally administrated $2 \%$ DSS for 7 days. The percentage of body weight changes (a) and survival rates (b) are shown. Data are shown as the mean \pm s.d. ${ }^{\star} P<0.05,{ }^{\star *} P<0.01$, and ${ }^{* * *} P<0.001$ by Student's $t$ test (a) or log-rank test (b). Hematoxylin and eosin staining of colon tissues at days 0 ( $n=4$ per group), 4 ( $n=7$ per group), and 8 ( $n=4$ per group) of DSS administration (c). The right panel shows the colitis score. Scale bar, $100 \mu \mathrm{m}$. Representative photos are shown. ${ }^{* \star *} P<0.001$ by Mann-Whitney test. (d) Immunoblot analysis of SRPRB, GRP78, and GNB2 in whole-cell lysates of colonic epithelial cells from 8-week-old Rnf186 ${ }^{+/+}$and $R n f 186^{-1-}$ mice at day 4 of DSS administration. Bottom, immunoblot analysis of actin (loading control). A representative blot of four independent experiments is shown. The right panel shows the densitometry of western blot signals with statistical evaluation. Data are shown as the mean \pm s.d. of four mice analyzed. ${ }^{*} P<0.05$ and ${ }^{* *} P<0.01$ by Student's $t$ test. DSS, dextran sulfate sodium; NS, not significant.

identified in the present study, the amount of occludin protein was increased in colonic epithelial cells and the distribution of occludin was altered in colonic epithelial cells in the absence of Rnf186. Tight junction molecules including occludin show dynamic protein behavior such as a continuous cycle of association and dissociation from tight junctions. ${ }^{33}$ The dynamic behavior of occludin is regulated by phosphorylation and ubiquitination. ${ }^{34}$ The present study showed that RNF186 maintains occludin homeostasis through ubiquitination in colonic epithelial cells. 
a

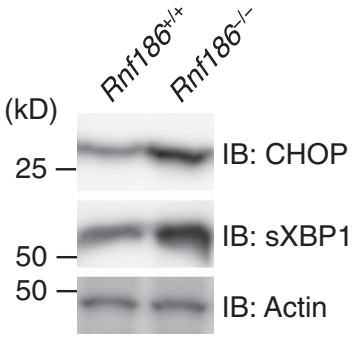

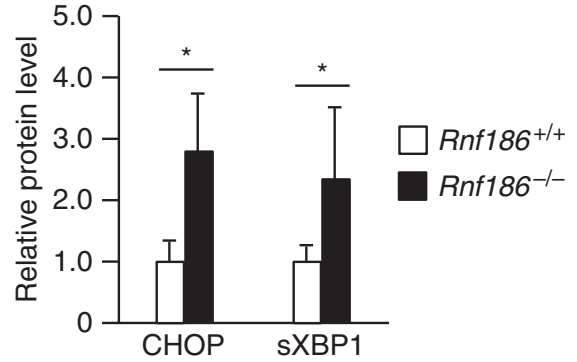

b

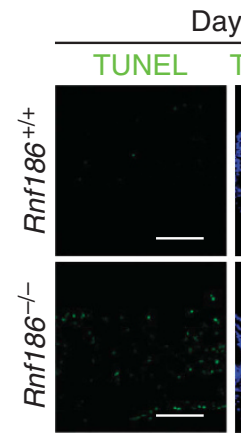

C
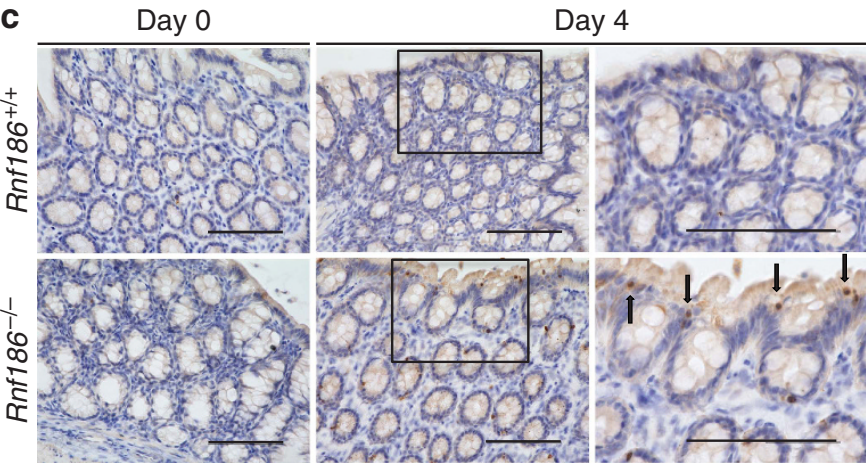

Day 4
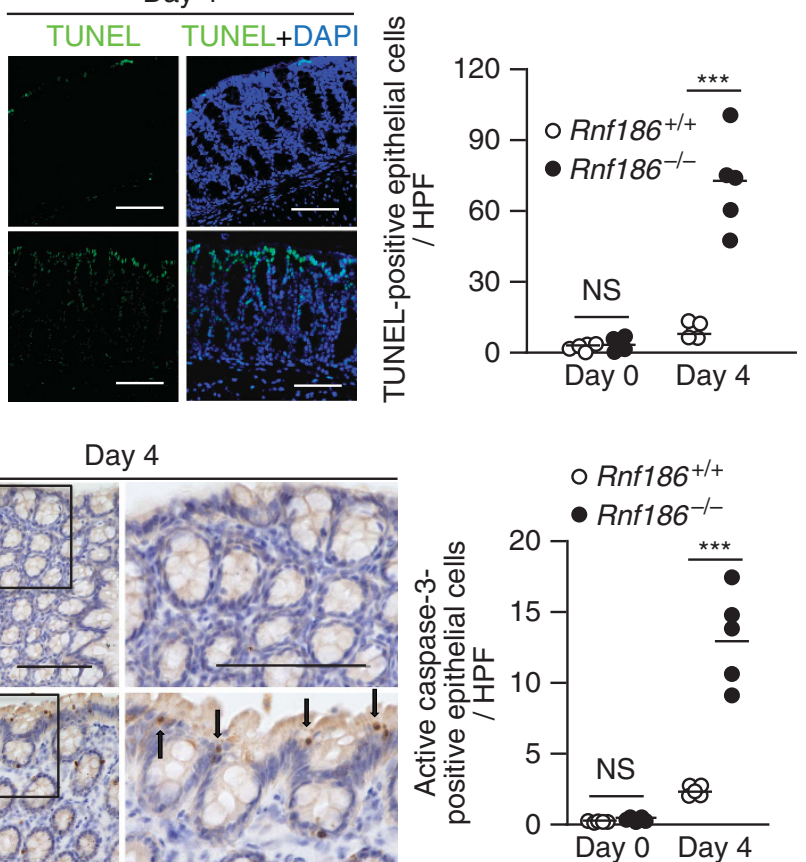

Figure 5 Enhanced ER stress in DSS-treated Rnf186 ${ }^{-1-}$ mice. (a) Immunoblot analysis of CHOP and SXBP1 in whole-cell lysates of colonic epithelial cells from 8-week-old $R n f 186^{+/+}$and $R n f 186^{-1-}$ mice at day 4 of DSS administration. Bottom, immunoblot analysis of actin (loading control). The right panel shows the densitometry of western blot signals with statistical evaluation. A representative blot of four independent experiments is shown. Data are shown as the mean \pm s.d. of four mice analyzed. ${ }^{*} P<0.05$ by Student's $t$ test. (b) TUNEL staining of colonic tissues in 8-week-old Rnf186 ${ }^{+/+}$and $R n f 186^{-1-}$ mice ( $n=5$ per group) at days 0 and 4 of DSS administration. TUNEL-stained colonic epithelial cells are indicated by green fluorescence. Nuclei were stained with DAPI (blue). Scale bar, $100 \mu \mathrm{m}$. Representative photos are shown. The right panel shows the numbers of TUNEL-positive epithelial cells. Each symbol represents an average of an individual mouse, and horizontal bars indicate the mean of five mice. ${ }^{* \star \star} P<0.001$ by Student's t test. (c) Immunohistochemical detection of active caspase-3 in colonic tissues of 8-week-old $R n f 186^{+/+}$and $R n f 186^{-1-}$ mice ( $n=5$ per group) at days 0 and 4 of DSS administration. Scale bar, $100 \mu \mathrm{m}$. Representative photos are shown. The right photos at days 4 show the boxed region at higher magnification. Black arrows show representative active caspase-3-positive epithelial cells. The right panel shows the numbers of active caspase-3positive epithelial cells. Each symbol represents an average of an individual mouse, and horizontal bars indicate the mean of five mice. ${ }^{* *} P<0.01$ by Student's $t$ test. DAPI, 4',6-diamidino-2-phenylindole; DSS, dextran sulfate sodium; NS: not significant.

Disturbances in proteostasis are involved in the pathogenesis of several disorders. ${ }^{31,32}$ Although $R n f 186^{-1-}$ mice did not develop colitis spontaneously, they showed increases in the permeability of organic solutes in the colon. This change might be due to the altered colonic distribution pattern of occludin, a component of tight junctions that mediate selective diffusion of organic solutes and ion solutions. ${ }^{35}$ However, a deficiency of occludin alone does not lead to the development of colonic inflammation. ${ }^{36}$ Indeed, another tight junction molecule, claudin-7, has been shown to regulate small organic solute diffusion in the colon and thereby regulates colonic homeostasis. ${ }^{37}$ Thus, an imbalance in homeostasis of proteins, including occludin and possibly other molecules associated with barrier functions, in colonic epithelial cells might affect the molecular dynamics of tight junction complexes, leading to increased permeability.

In addition to occludin, several RNF186 substrates were identified. Among them, SRPRB is one of the key factors for cotranslational protein translocation to the ER membrane..$^{38,39}$ The co-translational protein translocation pathway and the UPR work in a coordinated manner in the ER to maintain protein homeostasis during stress. ${ }^{40}$ Thus, insufficient degradation of SRPRB might secondarily contribute to the induction of dysregulated proteostasis in stressful condition. 


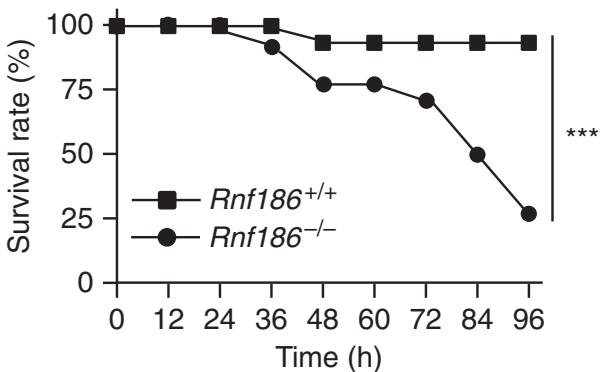

b

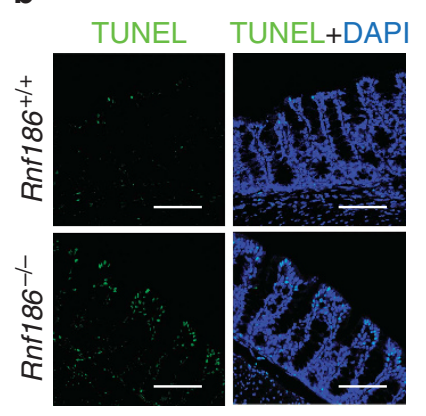

O Rnf186+/+

- Rnf186-/-

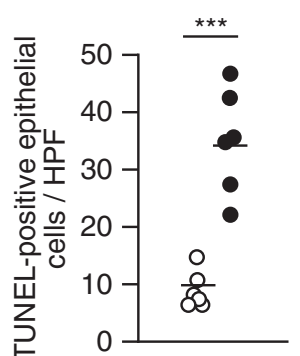

Figure 6 High sensitivity to tunicamycin-induced ER stress in $R n f 186^{-/-}$mice. (a) Eight- to twelve-week-old $R n f 186^{+/+}$( $n=13$ ) and $R n f 186^{-/-}$ $(n=18)$ mice were challenged by intraperitoneal injection of tunicamycin. The survival rates are shown. ${ }^{* \star *} P<0.001$ by log-rank test. (b) TUNEL staining of colonic tissues in $R n f 186^{+/+}$and $R n f 186^{-/}$mice ( $n=6$ per group) at $48 \mathrm{~h}$ after tunicamycin administration. Scale bar, $100 \mu$ m. Representative photos are shown. The right panel shows the numbers of TUNEL-positive epithelial cells. Each symbol represents an average of an individual mouse, and horizontal bars indicate the mean of six mice. ${ }^{* *} P<0.001$ by Student's $t$ test.

In the present study, disturbed protein homeostasis found in Rnf186 1 - colonic epithelia is discussed to be mediated by reduced protein degradation. However, it is possible that other mechanisms, such as enhanced protein stability and increased protein synthesis, contribute to the phenotype of $R n f 186^{-1-}$ mice. Future studies will be required to address the other mechanisms.

The present study showed that the disturbed proteostasis increases the ER stress in intestinal epithelial cells leading to the high sensitivity to DSS-induced intestinal inflammation. Similar findings have been reported in several studies. Mice treated with the proteasomal inhibitor MG132 are highly sensitive to DSS-induced intestinal inflammation. ${ }^{41}$ Mice lacking Xbp1, Mbtps1, Em2 (encoding IRE1b), or Oasis, all of which increase ER stress in intestinal epithelial cells, do not develop colitis spontaneously, but they are vulnerable to DSSinduced intestinal inflammation. ${ }^{15,16,26,42}$ Thus, an increase of ER stress in colonic epithelial cells increases the risk of intestinal inflammation. ${ }^{13}$ In addition, ER stress is reported to be induced in human IBD patients. Expression of GRP78 and sXBP1 is enhanced in the small and large intestines of CD patients. ${ }^{15,43}$ In UC patients, GRP78 expression is increased in the colon, especially in the inflamed mucosa. ${ }^{14,15,43}$ The ER stress observed in IBD patients may be induced secondarily to the intestinal inflammation. However, several mouse models of intestinal inflammation, such as mice lacking molecules in the UPR pathway (Xbp1, Mbtps1, and Em2), ${ }^{15,16,26}$ which are not able to cope with the ER stress, as well as mutant mice with aberrant protein production and subsequent enhanced ER stress, ${ }^{44}$ all show that ER stress triggers intestinal inflammation. The present study provides evidence that impaired protein degradation increases the risk of intestinal inflammation when colonic epithelial cells are exposed to stressful conditions.

In the present study, we showed that dysfunction of RNF186 leads to the enhanced ER stress and the increased risk of intestinal inflammation. However, because E3 ligases are involved in multiple cellular processes, it is possible that other mechanisms induced by the RNF186 dysfunction might contribute to the intestinal inflammation. Indeed, RNF186 has been shown to mediate enhanced ER stress responses. ${ }^{17}$ The discrepancy to our results would be due to different cell types used in the study. In addition, the signaling of LPS/IL-1mediated inhibitor of RXR functions was most significantly affected among down-regulated proteins in $R n f 186^{-1-}$ colonic epithelia, thus indicating the possible involvement of this pathway in the high sensitivity to intestinal inflammation. Therefore, it would be an interesting future issue to address whether other mechanisms are involved in the intestinal inflammation observed in the Rnf186 polymorphism.

Dysregulated proteostasis itself does not directly cause intestinal inflammation in $R n f 186^{-1-}$ mice. In this regard, as described above, several molecules involved in the UPR pathway might compensate for Rnf186 deficiency. Intestinal epithelial cells are continuously exposed to the luminal environment and should cope with ER stress induced by environmental factors. Therefore, in addition to protein degradation, intestinal epithelial cells possess several other mechanisms, including protein synthesis, maturation, glycosylation, and secretion, for the maintenance of proteostasis. A diet high in saturated fat has been shown to alter the intestinal environment, leading to an increased incidence of intestinal inflammation in $I l 10^{-1-}$ mice. ${ }^{45}$ Thus, it would be an interesting future direction to determine whether Rnf186 ${ }^{-1-}$ mice succumb to altered intestinal environments induced by a high fat diet.

The UC-associated variation (A64T) of RNF186 resulted in impaired E3 ligase activity in the human protein and increased sensitivity to DSS-induced intestinal inflammation in mice. Thus, the A64T mutation of RNF186 is functionally associated with UC pathogenesis, although it does not induce spontaneous inflammation in a SPF condition in mice. The RNF186 A64T variant was identified in North American and European UC patients $\left(P=8.69 \times 10^{-4}\right.$; odd ratio $\left.=1.49(1.17-1.90)\right){ }^{9}$ However, the A64T SNP in RNF186 was not found in 117 Japanese UC patients and 104 healthy individuals (our unpublished data), indicating that the non-synonymous coding variant in RNF186 is distinctly rare in UC patients and is 
unlikely to be a major primary cause of the UC pathogenesis. Differential frequencies of IBD-associated SNPs between Caucasian and Asian populations have also been reported for NOD2. ${ }^{46}$ IBD pathogenesis is governed by not only genetic factors, but also environmental factors. ${ }^{2}$ Intestinal environments, particularly the composition of microbiota, vary among populations depending on long-term dietary patterns. ${ }^{47}$ Thus, various intestinal environments have a significant effect on IBD pathogenesis, which might cause differential genetic susceptibility loci for IBD among distinct populations. Thus, IBD is a disorder induced by multiple and highly complicated factors. a

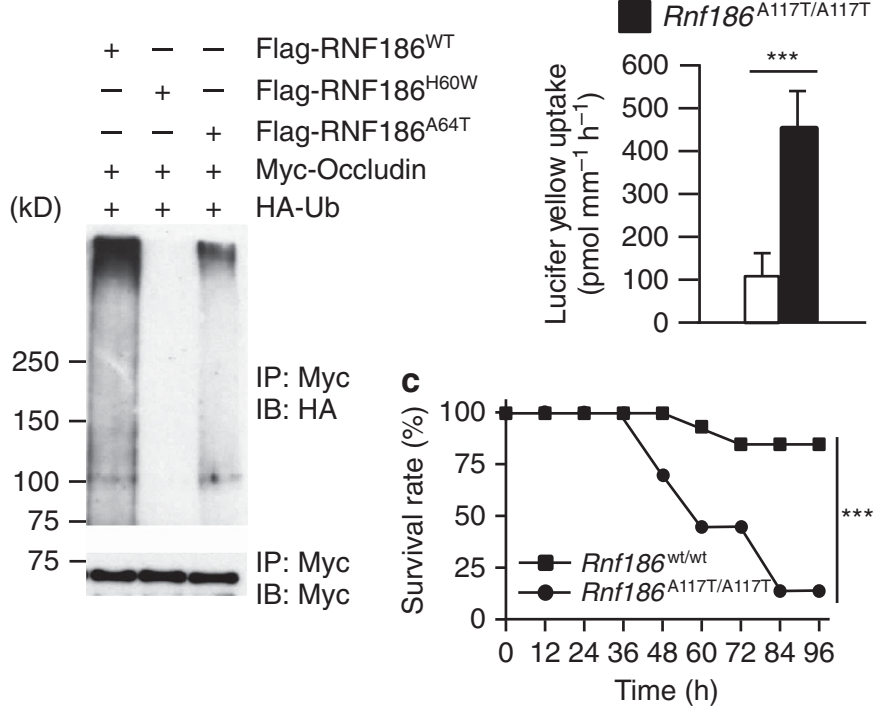

d
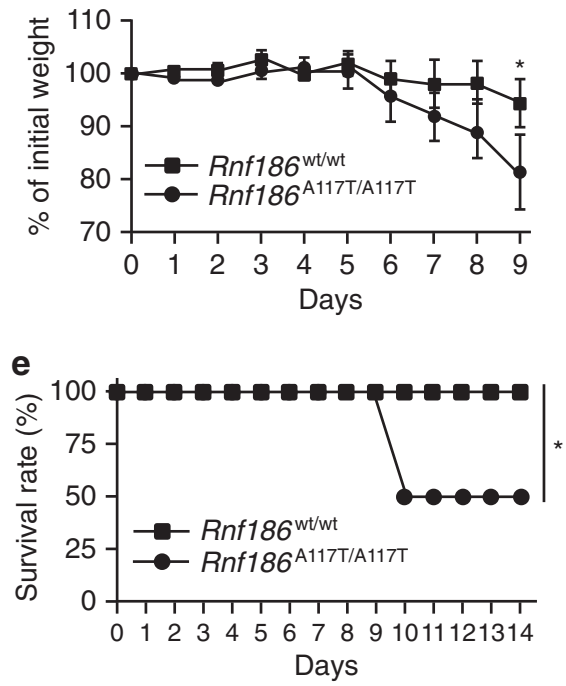

f
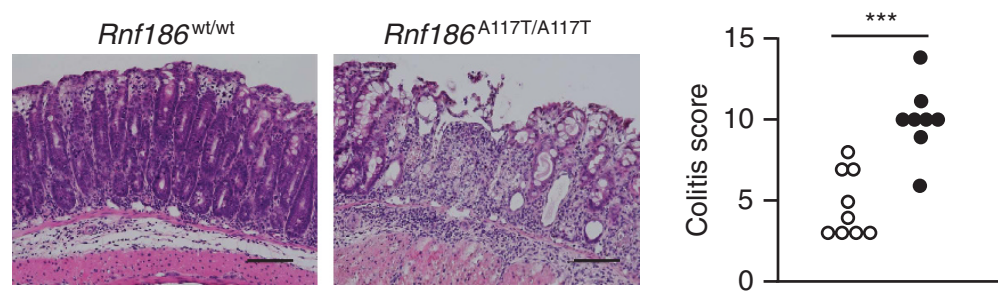

O Rnf186 $6^{\mathrm{w} / \mathrm{wt}}$

- Rnf186 $6^{\mathrm{A} 117 \mathrm{~T} / \mathrm{A} 117 \mathrm{~T}}$

g
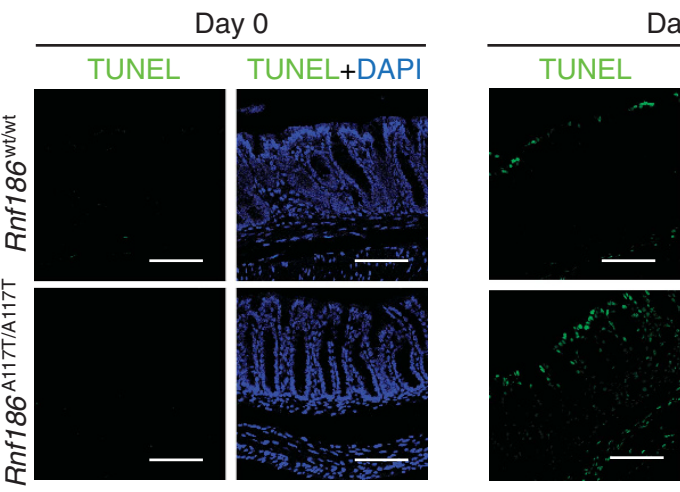

Day 5
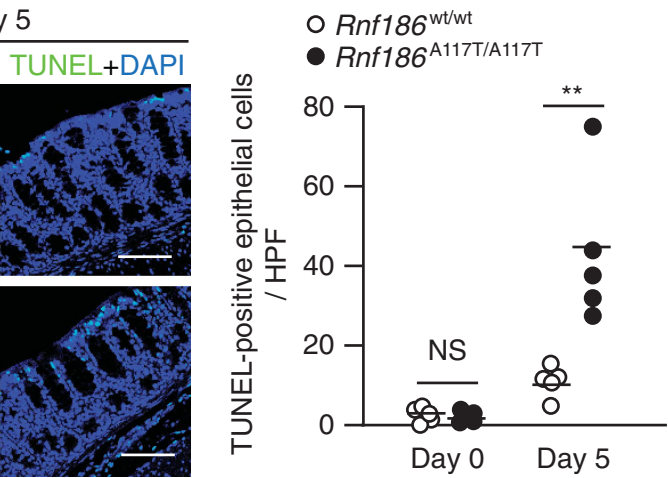

h
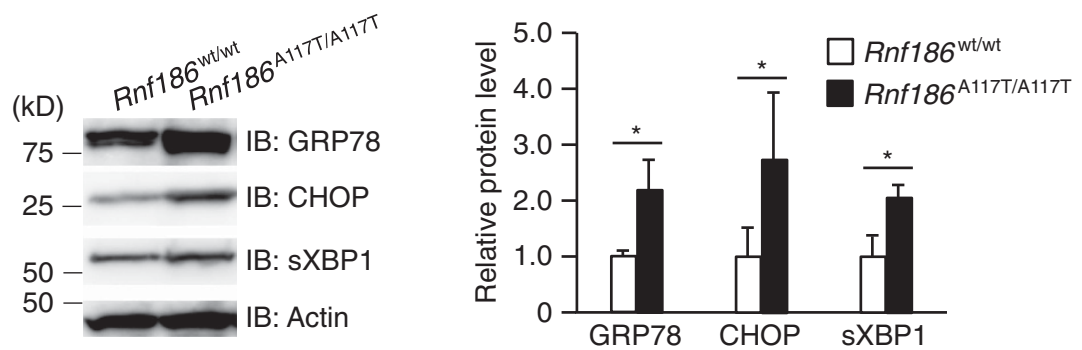
Our study revealed that the UC-associated gene RNF186 is functionally involved in the maintenance of gut homeostasis through controlling proteostasis in colonic epithelia. The A64T mutation of RNF186 results in a high risk for intestinal inflammation. Therefore, RNF186 may be a candidate marker for the diagnosis and prognosis of UC development.

\section{METHODS}

Mice. C57BL/6 J mice were purchased from Japan SLC (Shizuoka, Japan). Rnf186 ${ }^{-1-}$ mice and Rnf186 ${ }^{\mathrm{A} 117 \mathrm{~T} / \mathrm{A} 117 \mathrm{~T}}$ mice were generated as described in Supplementary Material. All mice were maintained under specific pathogen-free conditions at the Experimental Animal Facility of the Graduate School of Medicine, Osaka University. Animal experiments were performed according to guidelines of the Animal Research Committee of the Graduate School of Medicine at Osaka University.

Isolation of lamina propria and epithelium of large intestines. Large intestinal lamina propria and epithelium were isolated using previously described protocol. ${ }^{48}$ More precisely, large intestines of Rnf186 ${ }^{+}+$and $R n f 186^{-1-}$ mice were opened longitudinally and washed to remove fecal content in sterile phosphate-buffered saline (PBS). Large intestines were then incubated in sterile HBSS (Nacalai Tesque, Kyoto, Japan) containing $30 \mathrm{mM}$ EDTA at room temperature for $5 \mathrm{~min}$ with rigorous shaking to elute the epithelium from large intestines and the supernatant was centrifuged at 3,000 $\times \mathrm{g}$ for $5 \mathrm{~min}$. The resulting cell pellet was washed with ice-cold sterile PBS and used for epithelial cells. For isolation of lamina propria, after removing the epithelial layer, fat tissue was also removed from large intestines.

Quantitative RT-PCR. RNA samples were prepared from the indicated tissues using TRIzol reagent (Invitrogen, Yokohama, Japan). Total RNA was reverse transcribed using Moloney murine leukemia virus reverse transcriptase (Promega, Tokyo, Japan) and random primers (Toyobo, Osaka, Japan) after treatment with RQ1 DNase I (Promega). Complimentary DNA was analyzed by real-time RT-PCR using GoTaq qPCR Master Mix (Promega) in an ABI 7300 (Applied Biosystems, Yokohama, Japan). Values were normalized to the expression of Gapdh, and the fold difference in expression relative to Gapdh expression is shown. The primer sets are shown in Supplementary Table 2.

FITC-dextran permeability assay. Eight-week-old and one-year-old $R n f 186^{+1+}$ and $R n f 186^{-1-}$ mice were administrated with 4,400 Da FITC-Dextran (40 mg/100 g body weight) (Sigma-Aldrich, Tokyo, Japan) at $4 \mathrm{~h}$ before sacrifice. After obtaining whole blood, absorption of $100 \mu \mathrm{l}$ serum or the standard at $488 \mathrm{~nm}$ was measured in a fluorometer. As a standard curve, FITC-dextran was diluted in PBS.
Measurement of small organic solute uptake into the colon by an everted intestinal sac. The large intestines of 8 -week-old and 1 -year-old $R n f 186^{+I+}$ and $R n f 186^{-/-}$mice as well as 6-week-old Rnf186 $6^{\text {wt/wt }}$ and Rnf186 $6^{\text {A117T/A117T }}$ mice were isolated and the paracellular permeability was measured as described previously. ${ }^{37}$

Electrophysiological measurements. The middle colon of 8-week-old and 1-year-old Rnf186 ${ }^{+1+}$ and $R n f 186^{-1-}$ mice was isolated and the musculature was removed by blunt dissection. The sample was then mounted as a flat sheet between two Ussing chambers with an exposed area of $0.2 \mathrm{~cm}^{2}$ to examine the transepithelial conductance and the $\mathrm{NaCl}$ dilution potential. ${ }^{37}$

Plasmid construction. Plasmids were generated as described in Supplementary Material.

Immunoprecipitation and western blotting. HEK293T cells transfected with the indicated plasmids were lysed in lysis buffer containing $1.0 \%$ Nonidet P-40, $150 \mathrm{mM} \mathrm{NaCl}, 20 \mathrm{mM}$ Tris- $\mathrm{HCl}$ (pH 7.5), $5 \mathrm{mM}$ EDTA, and a protease inhibitor mixture. The lysates were sonicated for $5 \mathrm{~min}$ and then centrifuged at $13,000 \mathrm{~g}$ for $5 \mathrm{~min}$. To reduce nonspecific binding, the supernatants were incubated with protein $G$ agarose (Millipore, Dermstadt, Germany) at $4{ }^{\circ} \mathrm{C}$ for $30 \mathrm{~min}$. After centrifugation at $2,300 \mathrm{~g}$ for $5 \mathrm{~min}$, the supernatants were incubated with specific antibodies overnight at $4{ }^{\circ} \mathrm{C}$ followed by incubation with protein $\mathrm{G}$ agarose at $4{ }^{\circ} \mathrm{C}$ for $2 \mathrm{~h}$. After centrifugation at $2,300 \mathrm{~g}$ for $5 \mathrm{~min}$ and washing with $1 \mathrm{ml}$ lysis buffer four times, the immunoprecipitants were resolved by SDS-PAGE. The resolved proteins were transferred to a polyvinyl difluoride membrane (BIORAD, Hercules, CA) and blotted with the indicated antibodies. Immunoreactivity was detected using SuperSignal (Thermo Scientific, Yokohama, Japan).

Colon epithelial cells from the indicated mice were lysed and sonicated as described above. The lysates were centrifuged at $13,000 \mathrm{~g}$ for $5 \mathrm{~min}$ and the resulting supernatants were pre-incubated with protein $\mathrm{G}$ agarose at $4{ }^{\circ} \mathrm{C}$ for $30 \mathrm{~min}$. After centrifugation at $2,300 \mathrm{~g}$ for $5 \mathrm{~min}$, the supernatants were separated and transferred as describe above. The resolved proteins were blotted with the indicated antibodies. Immunoreactivity was detected using SuperSignal (Thermo Scientific). Digital analysis of the images was performed using ImageJ software (imagej.nih.gov/ij/).

LC-MS/MS analysis. Colon epithelial cells from 8-week-old Rnf186 ${ }^{+1+}$ and $R n f 186^{-1-}$ mice were lysed in lysis buffer containing $50 \mathrm{mM}$ Tris- $\mathrm{HCl}$ ( $\mathrm{pH} 7.6), 6 \mathrm{M}$ urea, $2 \%$ sodium deoxycholate, and a protease inhibitor mixture. The lysates were sonicated for $5 \mathrm{~min}$, centrifuged at $13,000 \mathrm{~g}$ for $5 \mathrm{~min}$, and the resulting supernatants were analyzed using Q-Exactive and UltiMate 3000 Nano LC systems (Thermo Fisher Scientific, Yokohama, Japan). ${ }^{49}$ Scaffold (version 4.4.5) was used to obtain a list of protein identifications with a false discovery rate $<0.0 \%$, requiring at least two individual peptides per protein with a

Figure 7 Association of the RNF186 A64T variant with intestinal inflammation. (a) Immunoblot analysis of anti-Myc immunoprecipitates from lysates of HEK293 cells co-transfected with the indicated expression vectors using anti-HA or anti-Myc antibodies. Data are representative of three independent experiments. (b) Rate of lucifer yellow uptake in the colons of 6-week-old $R n f 186^{\text {wt/wt }}(n=6)$ and $R n f 186^{\mathrm{A} 117 \mathrm{~T} / \mathrm{A} 117 \mathrm{~T}}(n=6)$ mice. Data are shown as the mean \pm s.d. ${ }^{* * \star} P<0.001$ by Student's $t$ test. (c) Eight- to twelve-week-old $R n f 186^{\text {wt }} /$ wt $(n=10)$ and $R n f 186^{\mathrm{A} 117 \mathrm{~T} / \mathrm{A} 117 \mathrm{~T}}(n=15)$ mice were challenged by intraperitoneal injection of tunicamycin. The survival rates are shown. ${ }^{\star \star \star} P<0.001$ by log-rank test. (d-f) Eight-week-old Rnf186 ${ }^{\text {wt/wt }}(n=7)$ and $\operatorname{Rnf186}^{\mathrm{A} 117 \mathrm{~T} / \mathrm{A} 117 \mathrm{~T}}(n=6)$ mice were orally administrated $2 \%$ DSS for 7 days. The percentage of body weight changes (d) and survival rates (e) are shown. Data are shown as the mean \pm s.d. ${ }^{*} P<0.05$ by Student's $t$ test (d) or log-rank test (e). Hematoxylin and eosin staining of colon tissues ( $n=8-9$ per group) at day 8 of DSS administration (f). The right panel shows the colitis score. Scale bar, $100 \mu \mathrm{m}$. Representative photos are shown. ${ }^{* * *} P<0.001$ by MannWhitney test. (g) TUNEL staining of colonic tissues in 8-week-old Rnf186 $6^{\mathrm{wt} / \mathrm{wt}}$ and Rnf186 $6^{\mathrm{A} 117 \mathrm{~T} / \mathrm{A} 117 \mathrm{~T}}$ mice $(n=5$ per group) at days 0 and 5 of DSS administration. TUNEL-stained colonic epithelial cells are indicated by green fluorescence. Nuclei were stained with DAPI (blue). Scale bar, $100 \mu \mathrm{m}$. Representative photos are shown. The right panel shows the numbers of TUNEL-positive epithelial cells. Each symbol represents an average of an individual mouse, and horizontal bars indicate the mean of five mice. ${ }^{* *} P<0.001$ by Student's $t$ test. (h) Immunoblot analysis of GRP78, CHOP, and sXBP1 in whole-cell lysates of colonic epithelial cells from 8-week-old Rnf186 ${ }^{\mathrm{wt} / \mathrm{wt}}$ and Rnf186 ${ }^{\mathrm{A} 117 \mathrm{~T} / \mathrm{A} 117 \mathrm{~T}}$ mice at day 6 of DSS administration. Bottom, immunoblot analysis of actin (loading control). A representative blot of three independent experiments is shown. The right panel shows the densitometry of western blot signals with statistical evaluation. Data are shown as the mean \pm s.d. of three mice analyzed. ${ }^{*} P<0.05$ by Student's $t$ test. DAPI, $4^{\prime}$, 6-diamidino-2-phenylindole; DSS, dextran sulfate sodium; NS: not significant. 
minimum peptide probability of $99.0 \%$. Overall, 2,665 proteins were identified and listed in Supplementary Table 1. Normalized total spectra, which are denoted as quantitative value in Scaffold, were used for quantitative comparison between colonic epithelial lysate data from $R n f 186^{+/+}$and $R n f 186^{-1-}$ mice ( $n=4$ per group). For statistical evaluation of the significance between $R n f 186^{+1+}$ and $R n f 186^{-1-}$ colonic epithelial proteins, moderated $t$ test, adjusted by theBenjaminiHochberg method, was performed using Scaffold and $P$ value of 0.05 was considered as a significant threshold. Similar quantitative proteomic analysis has been described previously. ${ }^{50}$ The volcano plot was created with Scaffold and the canonical pathway analysis and the functional analysis were generated through the use of QIAGEN's IPA (QIAGEN Redwood City, www.qiagen.com/ingenuity).

Induction of DSS-induced colitis. Eight-week-old $R n f 186^{-/-}$ mice and their $R n f 186^{+/+}$littermates as well as 8 -week-old Rnf186 ${ }^{\mathrm{A} 117 \mathrm{~T} / \mathrm{A} 117 \mathrm{~T}}$ mice and their $R n f 186^{\mathrm{wt} / \mathrm{wt}}$ littermates were used for DSS-induced colitis experiments. Acute colitis was induced by administration of $2 \%$ DSS in the drinking water for 7 days. Mice were analyzed for changes in body weight, survival rates, and histology.

Histological analysis. For whole-mount stain, colon tissues washed with solution $\mathrm{A}\left(120 \mathrm{mM} \mathrm{NaCl}, 10 \mathrm{mM} \mathrm{NaHCO}_{3}, 5 \mathrm{mM} \mathrm{KCl}, 1.2 \mathrm{mM}\right.$ $\mathrm{CaCl}_{2}$, and $10 \mathrm{mM}$ Tris-HEPES, $\mathrm{pH}$ 7.4). After removing muscle layers under the microscope, the tissues were fixed in 100\% methanol at $-20^{\circ} \mathrm{C}$ for $10 \mathrm{~min}$ and then washed with PBS. After blocking with $1 \%$ bovine serum albumin in PBS for $1 \mathrm{~h}$ at room temperature, the tissues were stained with anti-occludin for $1 \mathrm{~h}$ at $37^{\circ} \mathrm{C}$. The samples were washed and incubated with Alexa 488-conjugated anti-rat IgG and 4', 6-diamidino-2-phenylindole (DAPI) (cat\# D9564) (Sigma-Aldrich) for $1 \mathrm{~h}$ at $37^{\circ} \mathrm{C}$. The samples were washed with PBS, embedded in mounting medium (cat\# S3023) (Dako, Tokyo, Japan), and were observed with LSM-710 (Carl Zeiss, Tokyo, Japan). The images were analyzed with ZEN software (Carl Zeiss).

Indirect immunofluorescence was performed as described previously. ${ }^{37}$ Mouse intestines were frozen in liquid $\mathrm{N}_{2}$, and frozen sections $(5 \mu \mathrm{m})$ were prepared using a cryostat (Leica Japan), mounted on glass slides, air dried, and fixed in 100\% methanol at $-20{ }^{\circ} \mathrm{C}$ for $5 \mathrm{~min}$. The primary antibodies were anti-occludin. The second antibodies were Alexa 488-conjugated anti-rat IgG. Samples were examined using a BX-51 fluorescence microscope (Olympus, Tokyo, Japan).

Intestinal tissues from the indicated mice were collected and washed with PBS. After fixation in $4 \%$ paraformaldehyde overnight, the tissues were embedded in paraffin. Five-micrometer sections were stained with hematoxylin and eosin, and observed using a BZ-9000 microscope (Keyence, Osaka, Japan). Severity of colitis was evaluated by the standard scoring system as previously described. ${ }^{51}$ The scoring was performed blinded manner by pathologists.

Cell death in colonic tissues was determined by TUNEL staining. Paraffin-embedded sections of colonic tissues were treated with reagents supplied in an in situ Cell Death Detection Kit, Fluorescein (Roche Diagnostics, Tokyo, Japan) according to the manufacturer's protocol. The samples were stained with DAPI (cat\# 12745-74) (Nacalai Tesque) and observed under the confocal microscope. Numbers of TUNEL-positive colonic epithelial cells were counted in random ten high power microscopic fields (HPF) at $\times 40$ magnification. The calculated number in each mouse was statistically analyzed. $^{52}$

Paraffin-embedded sections of $R n f 186^{+/+}$and $R n f 186^{-/-}$ileum and colon were prepared as described above. To quench endogenous peroxidase activity, the sections were treated with $3 \%$ hydroperoxide for $10 \mathrm{~min}$ at room temperature. Then, the sections were heat treated in citrate buffer ( $\mathrm{pH} \mathrm{6}$ ) for $15 \mathrm{~min}$ at $121^{\circ} \mathrm{C}$ for antigen retrieval. After blocking with $10 \%$ goat serum in $\mathrm{PBS}$ for $1 \mathrm{~h}$ at room temperature, the sections were stained with anti-cleaved caspase-3 (1:100) antibodies or anti-lysozyme $(1: 100)$ antibodies overnight at $4{ }^{\circ} \mathrm{C}$. The sections were washed and incubated with biotin-conjugated goat anti-rabbit IgG
$(1: 2,000)$ for $1 \mathrm{~h}$ at $37^{\circ} \mathrm{C}$. After incubation with streptavidinconjugated horseradish peroxidase (Thermo Fisher Scientific) for $30 \mathrm{~min}$ at room temperature, the sections were visualized with DAB (3,3'-diaminobenzidine and hydroperoxide) (Wako, Osaka, Japan), stained with hematoxylin, and observed using a BZ-9000 microscope. Numbers of active caspase-3-positive colonic epithelial cells were counted in random four HPFs at $\times 40$ magnification. The calculated number in each mouse was statistically analyzed. ${ }^{52}$

Paraffin-embedded sections of $R n f 186^{+/+}$and $R n f 186^{-/-}$ileum were prepared as described above. The sections were heat treated in citrate buffer ( $\mathrm{pH} \mathrm{6}$ ) for $15 \mathrm{~min}$ at $121{ }^{\circ} \mathrm{C}$ for antigen retrieval. After blocking with $10 \%$ goat serum in PBS for $1 \mathrm{~h}$ at room temperature, the sections were stained with anti-MUC2 (1:100) antibodies for $1 \mathrm{~h}$ at room temperature. The sections were washed and incubated with Alexa 568-conjugated anti-rabbit $\operatorname{IgG}(1: 500)$ for $2 \mathrm{~h}$ at room temperature. The samples were stained with DAPI (cat\# 12745-74) (Nacalai Tesque) and observed under the confocal microscope.

In vivo UPR induction. Eight- to twelve-week-old $R n f 186^{-/-}$mice and their $R n f 186^{+/+}$littermates as well as $R n f 186^{\mathrm{Al17T} / \mathrm{A} 117 \mathrm{~T}}$ mice and their $R n f 186^{\mathrm{wt} / \mathrm{wt}}$ littermates were intraperitoneally injected with tunicamycin ( $2 \mathrm{mg} \mathrm{kg}^{-1}$ body weight) (Sigma-Aldrich) diluted in DMEM containing $3 \%$ fetal calf serum and $2 \%$ penicillin/streptomycin. Survival was monitored over $96 \mathrm{~h}$.

Statistical analysis. All statistical analyses were performed using StatMate version IV statistical software (ATMS, Tokyo, Japan), Scaffold (ver. 4.4.5), or IPA. A $P$ value of $<0.05$ was considered as statistically significant. Error bars denote the standard deviation.

SUPPLEMENTARY MATERIAL is linked to the online version of the paper at http://www.nature.com/mi

\section{ACKNOWLEDGMENTS}

We thank S. Nishioka (Nonprofit Organization Biotechnology Research and Development) for technical assistance in generating Rnf186 knock-in mice, N. Takemura, J. Ikeda and E. Morii for histological analysis, K. Takafuji for proteomics, S. Pareek for critical reading of the manuscript, T. Kondo and Y. Magota for technical assistance, and C. Hidaka for secretarial assistance. This work was supported by grants from the Ministry of Education, Culture, Sports, Science and Technology, the Japan Agency for Medical Research and Development, a Grant-in-Aid for JSPS Fellows, a Scholarship for Doctoral Students in Immunology, Project MEET at Osaka University Graduate School of Medicine and Mitsubishi Tanabe Pharma Corporation, and the Ministry of Health, Labour and Welfare.

\section{AUTHOR CONTRIBUTIONS}

K.F., M.K., and K. Takeda designed the study. K.F., M.K., A.T., H.T., Y.S., and Y.F. performed experiments. A.T., S.H., and S.T. provided reagents. H.K. and R.O. performed animal experiments. D.O. performed proteomics analysis. K.F. and M.I. generated Rnf186 knock-in mice. K. Tsuchiya and M.W. collected and provided human genomic DNA samples. M.N. and A.K. provided scientific insights. K. Takeda directed the research, and K.F. and K. Takeda wrote the paper.

\section{DISCLOSURE}

The authors declared no conflict of interest.

(c) 2017 Society for Mucosal Immunology

\section{REFERENCES}

1. Podolsky, D.K. Inflammatory bowel disease. N. Engl. J. Med. 347, 417-429 (2002).

2. Xavier, R.J. \& Podolsky, D.K. Unravelling the pathogenesis of inflammatory bowel disease. Nature 448, 427-434 (2007).

3. Khor, B., Gardet, A. \& Xavier, R.J. Genetics and pathogenesis of inflammatory bowel disease. Nature 474, 307-317 (2011). 
4. Jostins, L. et al. Host-microbe interactions have shaped the genetic architecture of inflammatory bowel disease. Nature 491, 119-124 (2012).

5. Liu, J.Z. et al. Association analyses identify 38 susceptibility loci for inflammatory bowel disease and highlight shared genetic risk across populations. Nat. Genet. 47, 979-986 (2015).

6. Silverberg, M.S. et al. Ulcerative colitis-risk loci on chromosomes 1 p36 and $12 \mathrm{q} 15$ found by genome-wide association study. Nat. Genet. 41, 216-220 (2009).

7. Juyal, G. et al. An investigation of genome-wide studies reported susceptibility loci for ulcerative colitis shows limited replication in north Indians. PloS One 6, e16565 (2011).

8. Yang, S.K. et al. Genome-wide association study of ulcerative colitis in Koreans suggests extensive overlapping of genetic susceptibility with Caucasians. Inflamm. Bowel Dis. 19, 954-966 (2013).

9. Beaudoin, M. et al. Deep resequencing of GWAS loci identifies rare variants in CARD9, IL23R and RNF186 that are associated with ulcerative colitis. PLoS Genet. 9, e1003723 (2013).

10. Deshaies, R.J. \& Joazeiro, C.A. RING domain E3 ubiquitin ligases. Annu. Rev. Biochem. 78, 399-434 (2009).

11. Nakamura, N. The role of the transmembrane RING Finger proteins in cellular and organelle function. Membranes 1, 354-393 (2011).

12. MacGurn, J.A., Hsu, P.C. \& Emr, S.D. Ubiquitin and membrane protein turnover: from cradle to grave. Annu. Rev. Biochem. 81, 231-259 (2012).

13. Kaser, A., Martinez-Naves, E. \& Blumberg, R.S. Endoplasmic reticulum stress: implications for inflammatory bowel disease pathogenesis. Curr. Opin. Gastroenterol. 26, 318-326 (2010).

14. Heazlewood, C.K. et al. Aberrant mucin assembly in mice causes endoplasmic reticulum stress and spontaneous inflammation resembling ulcerative colitis. PLoS Med. 5, e54 (2008).

15. Kaser, A. et al. XBP1 links ER stress to intestinal inflammation and confers genetic risk for human inflammatory bowel disease. Cell 134, 743-756 (2008).

16. Brandl, K. et al. Enhanced sensitivity to DSS colitis caused by a hypomorphic Mbtps1 mutation disrupting the ATF6-driven unfolded protein response. Proc. Natl. Acad. Sci. U S A 106, 3300-3305 (2009).

17. Wang, P., Wu, Y., Li, Y., Zheng, J. \& Tang, J. A novel RING finger E3 ligase RNF186 regulate ER stress-mediated apoptosis through interaction with BNip1. Cell. Signal. 25, 2320-2333 (2013).

18. Darsigny, M. et al. Loss of hepatocyte-nuclear-factor-4alpha affects colonic ion transport and causes chronic inflammation resembling inflammatory bowel disease in mice. PloS One 4, e7609 (2009).

19. McGovern, D.P. et al. Genome-wide association identifies multiple ulcerative colitis susceptibility loci. Nat. Genet. 42, 332-337 (2010).

20. Tsukita, S., Furuse, M. \& Itoh, M. Multifunctional strands in tight junctions. Nat. Rev. Mol. Cell Biol. 2, 285-293 (2001).

21. Turner, J.R. Intestinal mucosal barrier function in health and disease. Nat. Rev. Immunol. 9, 799-809 (2009).

22. Traweger, A. et al. The tight junction-specific protein occludin is a functional target of the E3 ubiquitin-protein ligase itch. J. Biol. Chem. 277, 10201-10208 (2002).

23. Lee, S.J. et al. E3 ligase activity of RING finger proteins that interact with Hip-2, a human ubiquitin-conjugating enzyme. FEBS Lett. 503, 61-64 (2001).

24. Okumura, F., Yoshida, K., Liang, F. \& Hatakeyama, S. MDA-9/syntenin interacts with ubiquitin via a novel ubiquitin-binding motif. Mol. Cell Biochem. 352, 163-172 (2011).

25. Cao, S.S. et al. The unfolded protein response and chemical chaperones reduce protein misfolding and colitis in mice. Gastroenterology 144 , 989-1000 e1006 (2013).

26. Bertolotti, A. et al. Increased sensitivity to dextran sodium sulfate colitis in IRE1beta-deficient mice. J. Clin. Invest. 107, 585-593 (2001).

27. Ron, D. \& Walter, P. Signal integration in the endoplasmic reticulum unfolded protein response. Nat. Rev. Mol. Cell Biol. 8, 519-529 (2007).

28. Oyadomari, S. \& Mori, M. Roles of CHOP/GADD153 in endoplasmic reticulum stress. Cell Death Differ. 11, 381-389 (2004).
29. Wirtz, S., Neufert, C., Weigmann, B. \& Neurath, M.F. Chemically induced mouse models of intestinal inflammation. Nat. Protoc. 2, 541-546 (2007).

30. Inui, M. et al. Rapid generation of mouse models with defined point mutations by the CRISPR/Cas9 system. Sci. Rep. 4, 5396 (2014).

31. Balch, W.E., Morimoto, R.I., Dillin, A. \& Kelly, J.W. Adapting proteostasis for disease intervention. Science 319, 916-919 (2008).

32. Powers, E.T., Morimoto, R.I., Dillin, A., Kelly, J.W. \& Balch, W.E. Biological and chemical approaches to diseases of proteostasis deficiency. Annu. Rev. Biochem. 78, 959-991 (2009).

33. Shen, L., Weber, C.R. \& Turner, J.R. The tight junction protein complex undergoes rapid and continuous molecular remodeling at steady state. J. Cell Biol. 181, 683-695 (2008).

34. Murakami, T., Felinski, E.A. \& Antonetti, D.A. Occludin phosphorylation and ubiquitination regulate tight junction trafficking and vascular endothelial growth factor-induced permeability. J. Biol. Chem. 284, 21036-21046 (2009).

35. Tamura, A. \& Tsukita, S. Paracellular barrier and channel functions of TJ claudins in organizing biological systems: advances in the field of barriology revealed in knockout mice. Semin. Cell Dev. Biol. 36, 177-185 (2014).

36. Saitou, M. et al. Complex phenotype of mice lacking occludin, a component of tight junction strands. Mol. Biol. Cell. 11, 4131-4142 (2000).

37. Tanaka, H. et al. Intestinal deletion of Claudin-7 enhances paracellular organic solute flux and initiates colonic inflammation in mice. Gut 64, 1529-1538 (2015).

38. Schnell, D.J. \& Hebert, D.N. Protein translocons: multifunctional mediators of protein translocation across membranes. Cell 112, 491-505 (2003).

39. Rapoport, T.A. Protein translocation across the eukaryotic endoplasmic reticulum and bacterial plasma membranes. Nature 450, 663-669 (2007).

40. Plumb, R., Zhang, Z.R., Appathurai, S. \& Mariappan, M. A functional link between the co-translational protein translocation pathway and the UPR. Elife 4, e07426 (2015).

41. Inoue, S. et al. The effect of proteasome inhibitor MG132 on experimental inflammatory bowel disease. Clin. Exp. Immunol. 156, 172-182 (2009).

42. Hino, K., Saito, A., Asada, R., Kanemoto, S. \& Imaizumi, K. Increased susceptibility to dextran sulfate sodium-induced colitis in the endoplasmic reticulum stress transducer OASIS deficient mice. PloS One 9, e88048 (2014).

43. Shkoda, A. et al. Interleukin-10 blocked endoplasmic reticulum stress in intestinal epithelial cells: impact on chronic inflammation. Gastroenterology 132, 190-207 (2007).

44. Sitia, R. \& Braakman, I. Quality control in the endoplasmic reticulum protein factory. Nature 426, 891-894 (2003).

45. Devkota, S. et al. Dietary-fat-induced taurocholic acid promotes pathobiont expansion and colitis in II10 - / - mice. Nature 487, 104-108 (2012).

46. Inoue, N. et al. Lack of common NOD2 variants in Japanese patients with Crohn's disease. Gastroenterology 123, 86-91 (2002).

47. Wu, G.D. et al. Linking long-term dietary patterns with gut microbial enterotypes. Science 334, 105-108 (2011).

48. Kusu, T. et al. Ecto-nucleoside triphosphate diphosphohydrolase 7 controls Th17 cell responses through regulation of luminal ATP in the small intestine. J. Immunol. 190, 774-783 (2013).

49. Meissner, F. \& Mann, M. Quantitative shotgun proteomics: considerations for a high-quality workflow in immunology. Nat. Immunol. 15, 112-117 (2014).

50. Flenkenthaler, F. et al. Secretome analysis of testicular peritubular cells: a window into the human testicular microenvironment and the spermatogonial stem cell niche in man. J. Proteome Res. 13, 1259-1269 (2014).

51. Kobayashi, M. et al. Toll-like receptor-dependent production of IL-12p40 causes chronic enterocolitis in myeloid cell-specific Stat3-deficient mice. J. Clin. Invest. 111, 1297-1308 (2003).

52. Rhee, L. et al. Expression of TNFAIP3 in intestinal epithelial cells protects from DSS- but not TNBS-induced colitis. Am. J. Physiol. Gastrointest. Liver Physiol. 303, G220-G227 (2012). 